\title{
Immunological and Clinical Benefits of Maternal Immunization Against Pertussis: A Systematic Review
}

Charlotte Switzer · Caroline D'Heilly · Denis Macina

Received: May 8, 2019 / Published online: September 18, 2019

(C) The Author(s) 2019

\begin{abstract}
Infants are vulnerable to pertussis infection particularly before initiation of pertussis vaccination. Maternal pertussis vaccination during pregnancy has been introduced in a number of countries in order to confer on young infants indirect protection from the disease through transplacental transfer of maternal antibodies. We reviewed the evidence on the immunogenicity and efficacy of maternal pertussis vaccination during pregnancy. A systematic search of PubMed/MEDLINE, EMBASE, Scopus, Cochrane Database of Systematic Reviews, ProQuest, and Science Direct was undertaken to identify studies published between January 1995 and December 2018. This review was not specific to any particular pertussis vaccine but included applicable data on available pertussis vaccines administered to pregnant women. The search identified 40 publications for inclusion in this review. Vaccination during pregnancy elicited robust maternal immune responses against
\end{abstract}

Enhanced Digital Features To view enhanced digital features for this article go to https://doi.org/10.6084/ m9.figshare.9742709.

C. Switzer

Sanofi Pasteur, Pharmacoepidemiology, Toronto,

Canada

C. D’Heilly · D. Macina $(\bowtie)$

Sanofi Pasteur, Vaccines Epidemiology and

Modeling, Lyon, France

e-mail: denis.macina@sanofi.com all vaccine antigens and resulted in high placental transfer of pertussis antibodies to the infant that persisted well beyond delivery. Vaccination during the second or early third trimesters was considered ideal for antibody quantity and functionality. Although blunting of immune responses to some antigens in the primary immunization series was documented in neonates born to women vaccinated during pregnancy, there was no apparent adverse effect on vaccine efficacy. Multiple studies conducted in diverse settings have confirmed the effectiveness of maternal pertussis vaccination during pregnancy in preventing pertussis in infants prior to receipt of their first primary vaccine dose and beyond. These findings collectively underscore the value of maternal pertussis vaccination during pregnancy in protecting vulnerable infants too young to be vaccinated.

Funding Sanofi Pasteur.

Plain Language Summary Plain language summary available for this article.

Keywords: Efficacy; Immunization; Pertussis; Pregnancy

\section{PLAIN LANGUAGE SUMMARY}

Infants need protection against whooping cough particularly in the first few months after birth. Vaccination of women during pregnancy 
is recommended in some countries to protect the infant against the disease through the transfer of the mother's protective antibodies. We reviewed the published evidence on the immunological and clinical benefits of maternal vaccination against whooping cough. Vaccination of pregnant women elicited robust maternal immune responses against whooping cough and resulted in high antibody levels transferred to the infant. Infants born to women who were vaccinated during pregnancy were better protected against whooping cough in the first few months after birth than those whose mothers were not vaccinated. The best time to vaccinate pregnant women was in the late second or early third trimesters. Although the infant's immune responses to their primary immunizations were reduced in those born to women vaccinated during pregnancy, this did not affect vaccine effectiveness. This review confirms that maternal vaccination against whooping cough during pregnancy protects infants against the disease.

\section{INTRODUCTION}

Pertussis, whooping cough, results from infection of the upper respiratory tract by Bordetella pertussis. Infection resulting in at least mild disease was universal among children before the introduction of pertussis vaccination. Disease outbreak cycles occurred every 3-5 years, which primed most individuals through repeated exposure, and provided ongoing immunity after the first episode. Young infants were partially spared through indirect protection from maternal antibodies acquired in utero. Although routine pertussis vaccination of infants and young children reduced the incidence by more than 99\% [1], the disease still persists today. In addition, there has been a shift in the age-specific disease profile with, in particular, an increased proportion of cases among infants aged $<1$ year, adolescents and the elderly [2-7]. This age-specific shift has been attributed to waning of vaccine-induced immunity, and, in young infants, reduced pathogen circulation limiting natural boosting during pregnancy and subsequent passive protection. Infants (in particular those too young to have received the primary immunization series) have the highest rates of pertussis-related morbidity and mortality [6, 8-13].

Older siblings and adult close contacts are common reservoirs for pertussis transmission to young infants [14-16]. Strategies introduced to increase herd immunity have included booster doses for adolescents and close-contact adults. However, widening coverage with pertussis vaccines appears to have had little impact in preventing transmission to young infants or on the resurgence of periodic epidemic peaks in some countries in recent years [11, 13, 17]. Concerns that close contacts continue to remain common reservoirs for disease transmission to young infants have led to vaccination against pertussis during pregnancy [18-23]. Vaccination during pregnancy protects the mother directly, and, more importantly, provides passive protection (via transplacental antibody transfer in utero) for their young infants.

A number of clinical studies assessing the immunogenicity, efficacy and safety of maternal pertussis vaccination during pregnancy have recently been published. As such, it is timely to collate the available evidence and summarize the benefit-risk profile of pertussis vaccination during pregnancy. This will help informed decision-making and identify important gaps in the evidence that may require further investigation. This systematic review focuses on the immunological response to maternal pertussis vaccination during pregnancy as well as on vaccine efficacy in preventing infant pertussis. Maternal immunological responses, placental transfer of antibodies and infant responses to the primary immunization series are summarized. The safety of pertussis vaccination during pregnancy is the focus of an accompanying systematic review (D'Heilly et al., in preparation).

\section{METHODS}

We conducted a systematic review of the English language literature, following the PRISMA framework [24], to identify studies reporting the immunogenicity and efficacy of pertussis vaccination (tetanus toxoid, reduced-dose 
diphtheria toxoid, and reduced-dose acellular pertussis [Tdap] vaccine and Tdap-inactivated polio vaccine [IPV]) during pregnancy. The review protocol was registered on PROSPERO (PROSPERO 2016:CRD42016038317) [25].

Our search initially encompassed the period from January 1995 to June 2016, and was later updated to capture relevant studies published between July 2016 and October 2018. Databases searched included PubMed/MEDLINE, EMBASE, Scopus (Elsevier), Cochrane Database of Systematic Reviews, ProQuest, and Science Direct. Reference lists of identified publications were also scanned. ProQuest thesis, Clinicaltrials.gov, Conferences and Congresses, and Trial Trove were searched for unpublished evidence. The search algorithms used were [1]: [(pertussis OR whooping cough) AND (vaccine OR Tdap OR immunization) AND (pregnancy OR pregnant OR pre-partum OR gestation OR maternal)], and [2]: [1] AND (immunogenicity OR immunity OR immune response OR antibody OR antibodies OR effectiveness OR efficacy).

Interventional and observational studies in humans were considered using the Patient Population, Intervention, Comparison, Outcomes, and Setting criteria [26]. The population was pregnant women and their offspring; the interventions were pertussis immunization during pregnancy and the infant series of vaccination; comparison groups were either no vaccination or standard-ofcare vaccination, as well as pregnancy or no pregnancy; the outcomes were absolute and relative immunogenicity measures against pertussis vaccine antigens in maternal, umbilical cord, and/or infant blood, as well as infant pertussis antibody responses following each dose of the primary and toddler booster vaccination course, qualitative (clinical symptoms, severity) and quantitative [number, frequencies and relative (e.g., OR, RR)] disease outcome measures, including vaccine efficacy and/or vaccine effectiveness estimates. We excluded reviews, case reports, opinions and letters to editors, meta-analyses, modeling studies, and studies on vaccination program improvement, vaccine uptake increase or health economics, and vaccine acceptability and perception studies.

Selection of publications for inclusion was done in two steps, with two independent reviewers involved at each step. Titles and abstracts of retrieved publications were first screened for relevance. Publications documenting primary research (excluding modeling studies), and specifically dealing with outcomes of pre-partum pertussis vaccination, were retained for full-text review to determined compliance with inclusion and exclusion criteria. Relevant data from included studies were extracted using pre-structured MS Excel forms. Where possible, vaccine brand names are presented to help ascertain the number of pertussis components assessed. A meta-analysis of the immunologic or efficacy/effectiveness findings was not feasible because of heterogeneity in the study designs, vaccines used, measured outcomes, measurement/analysis methods, and background pertussis incidence rates.

This article is based on previously conducted studies and does not contain any studies with human participants or animals performed by any of the authors.

\section{RESULTS}

A total of 293 articles were identified, of which 43 articles (summarized in Tables 1 and 2, respectively) were included in this review (Fig. 1).

\section{Immune Indicators}

Maternal immune responses to pertussis antigens in both the mother and child were considered in this review. However, extrapolation of persisting antibody geometric mean concentrations (GMCs) to protection against pertussis is not possible as there is no agreed immunological correlate of protection.

\section{Maternal Immune Responses}

Vaccination with Tdap during pregnancy induces a robust maternal immune response (Table 1) [27-40], irrespective of maternal body mass index [30]. Pertussis antibody levels measured $\geq 1$ month after Tdap immunization, or at delivery, were substantially higher against all vaccine antigens [pertussis toxin (PT), filamentous hemagglutinin (FHA), pertactin, and 


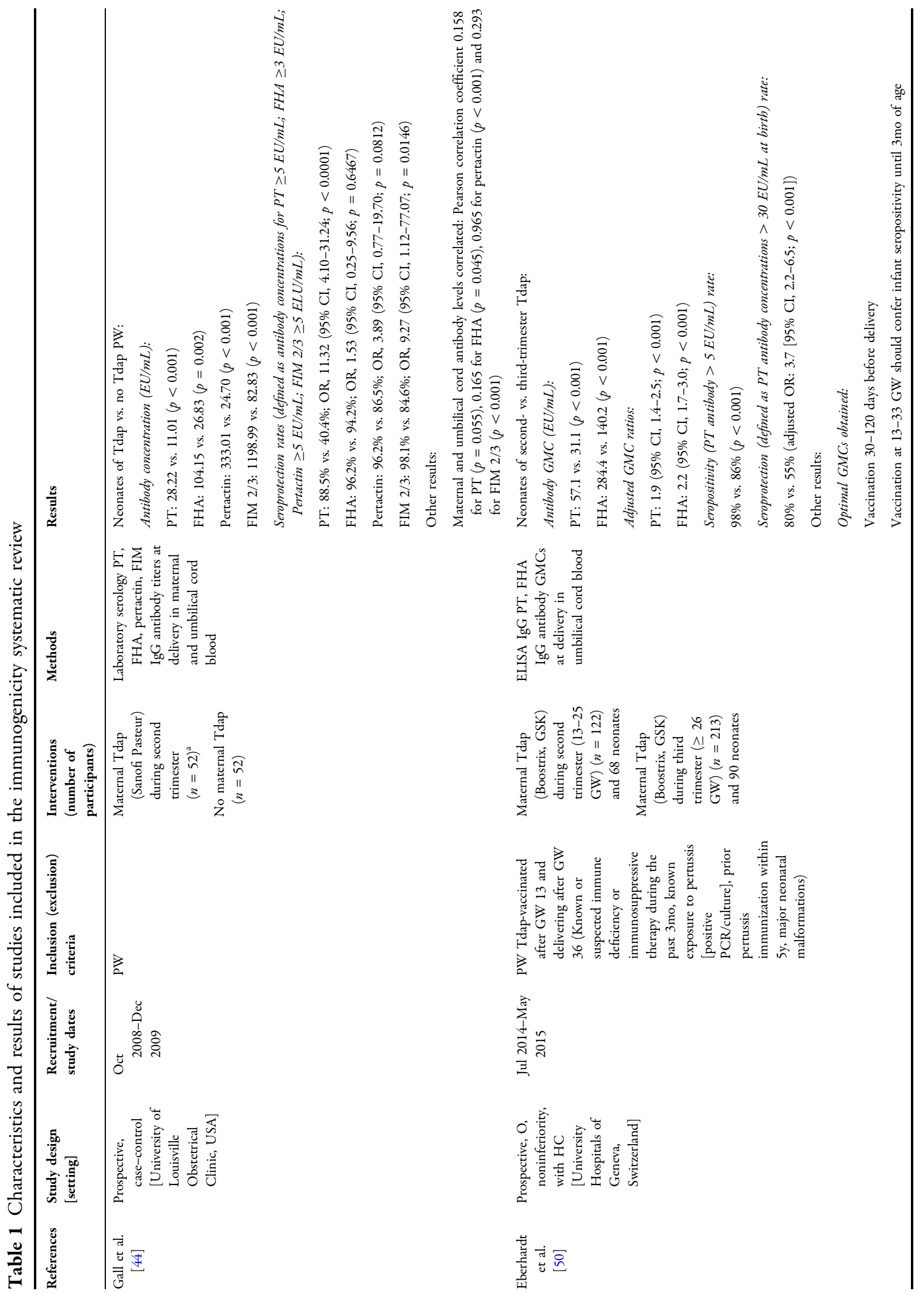




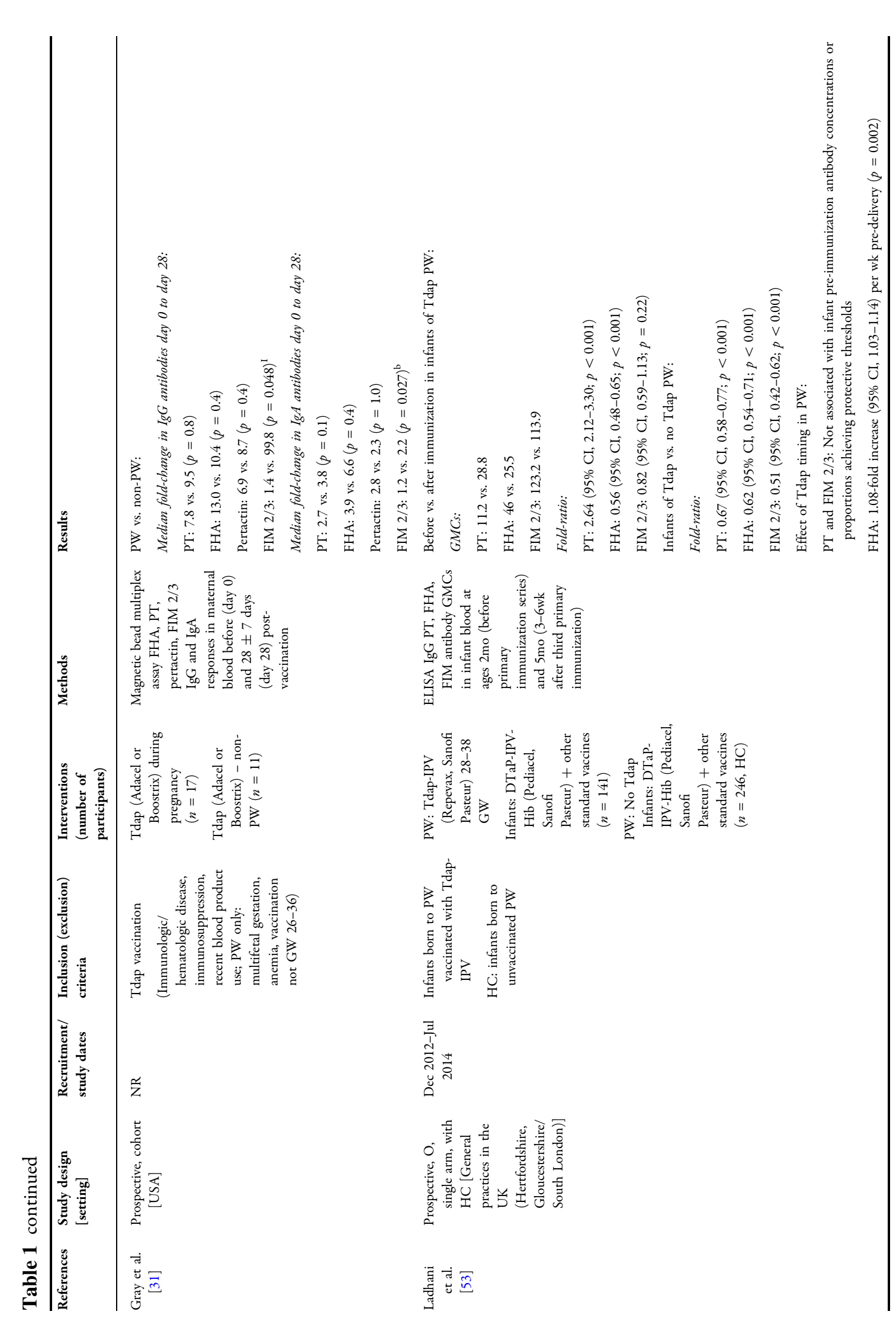




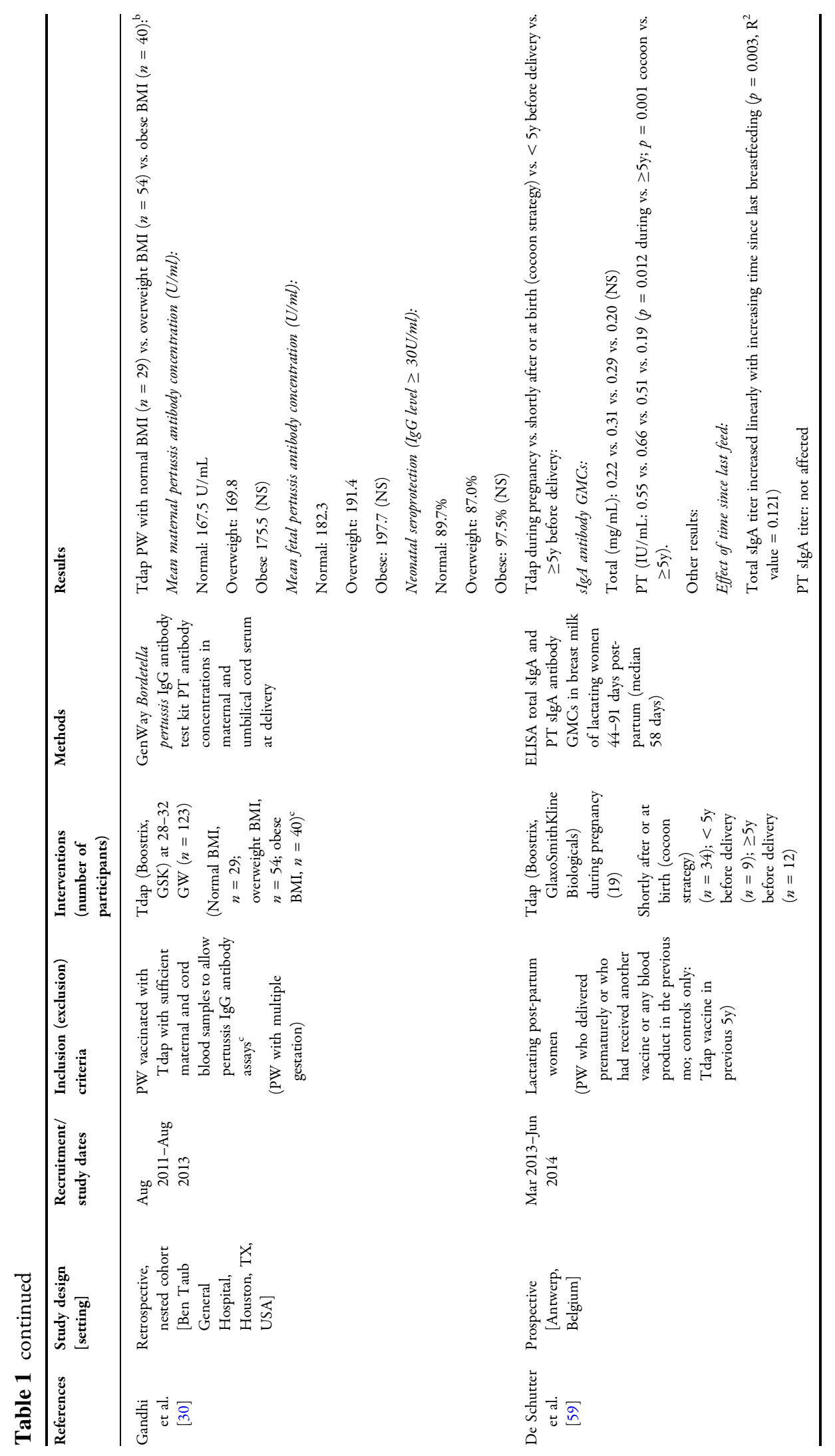




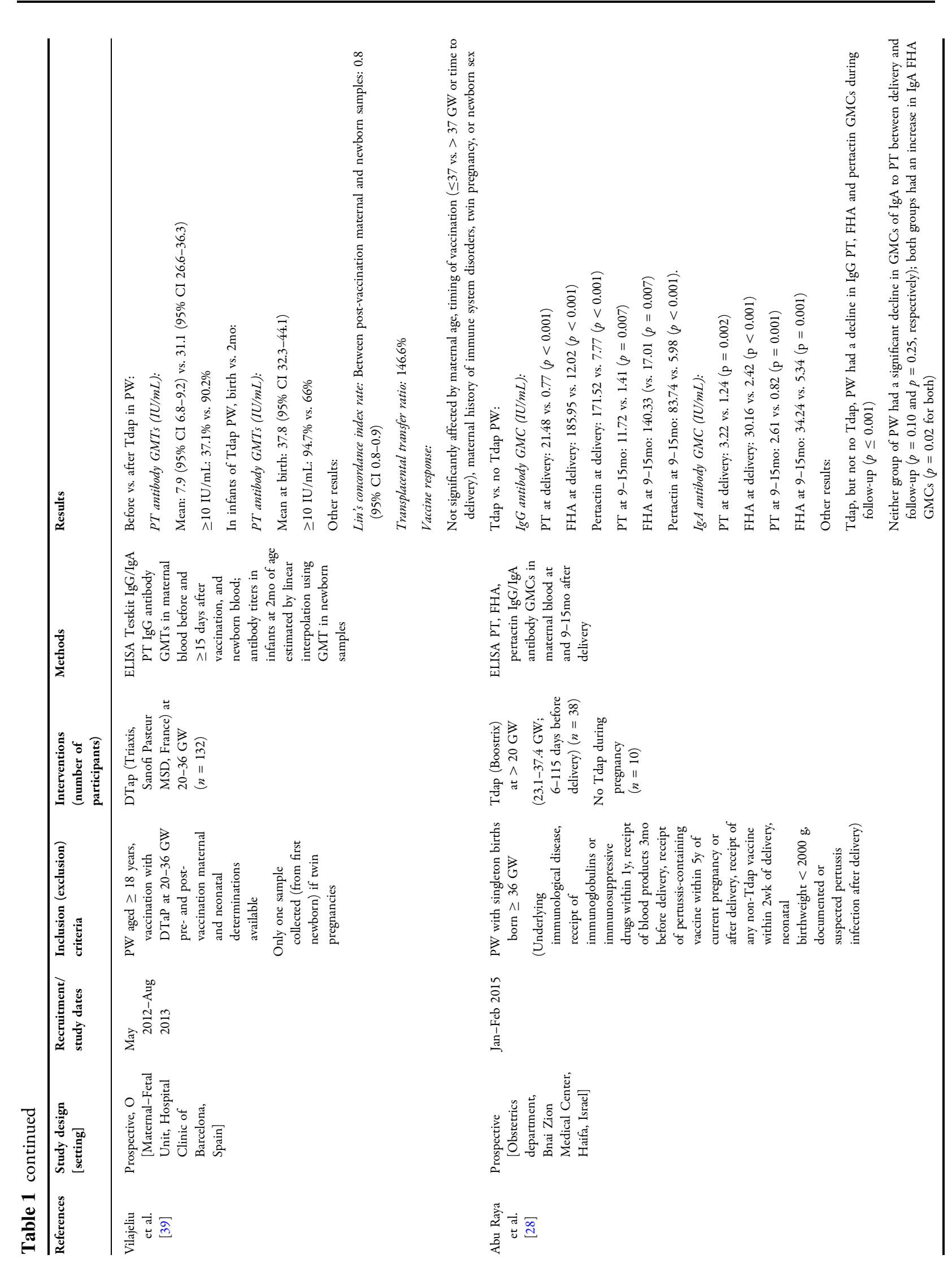




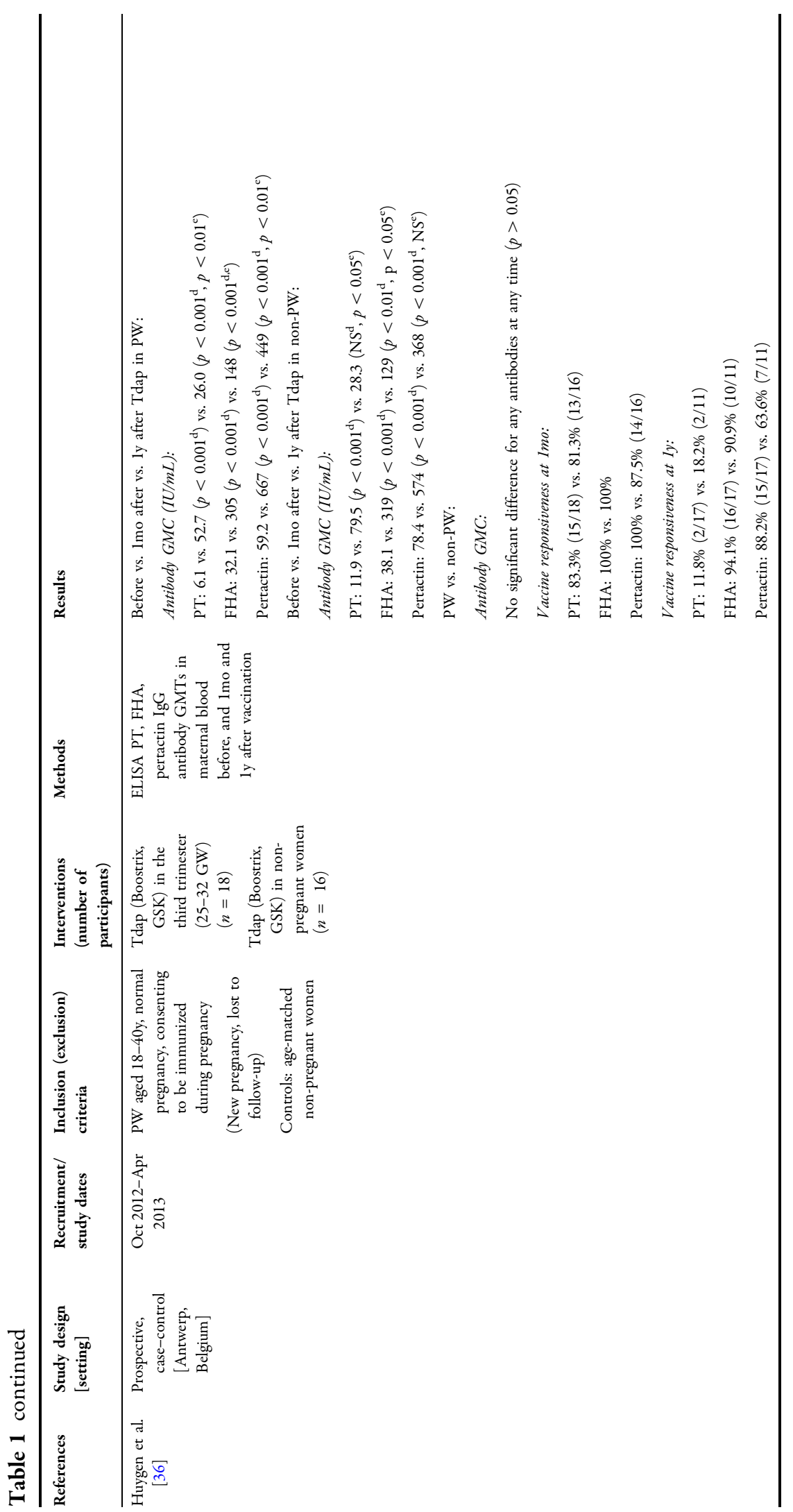




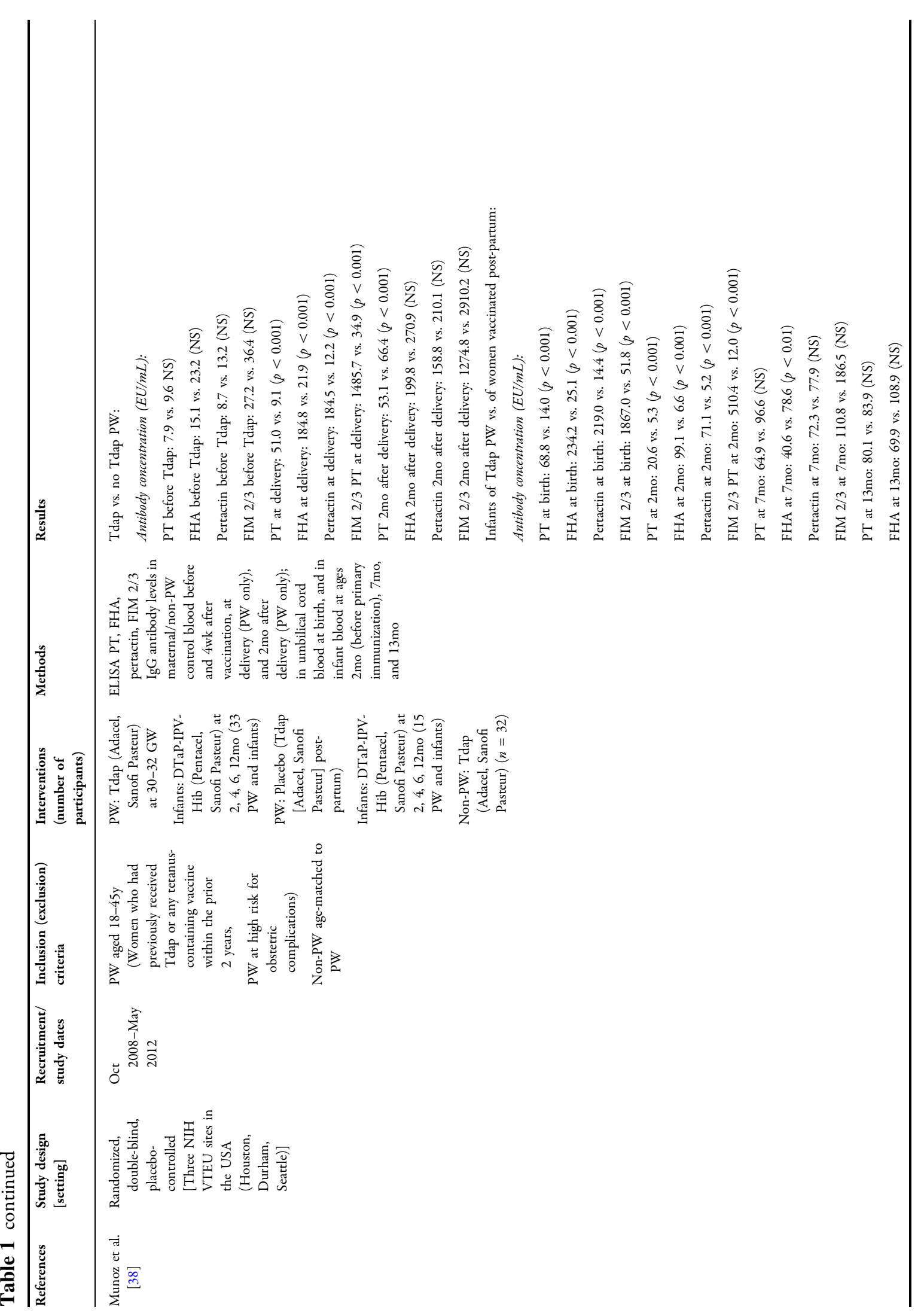




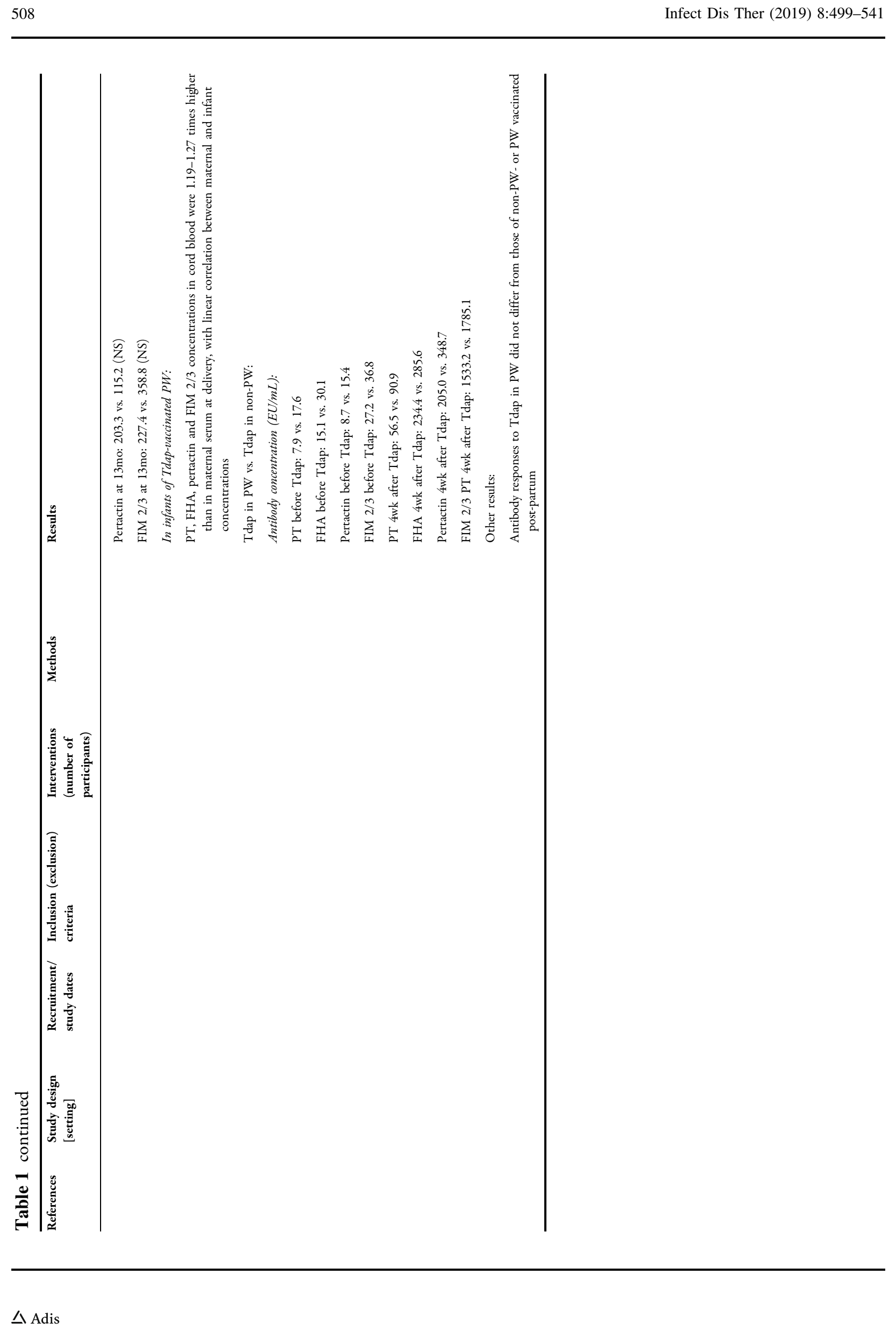




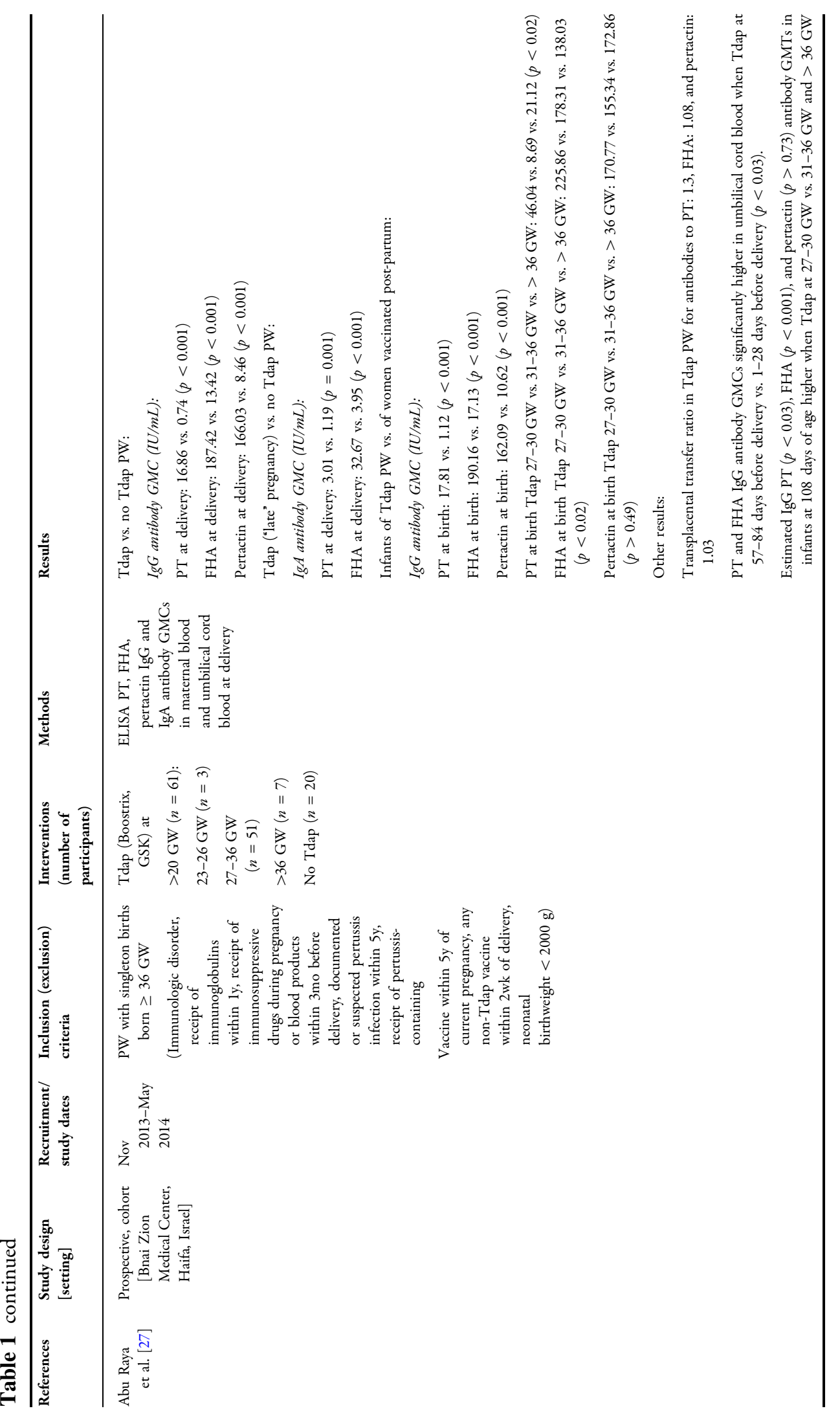




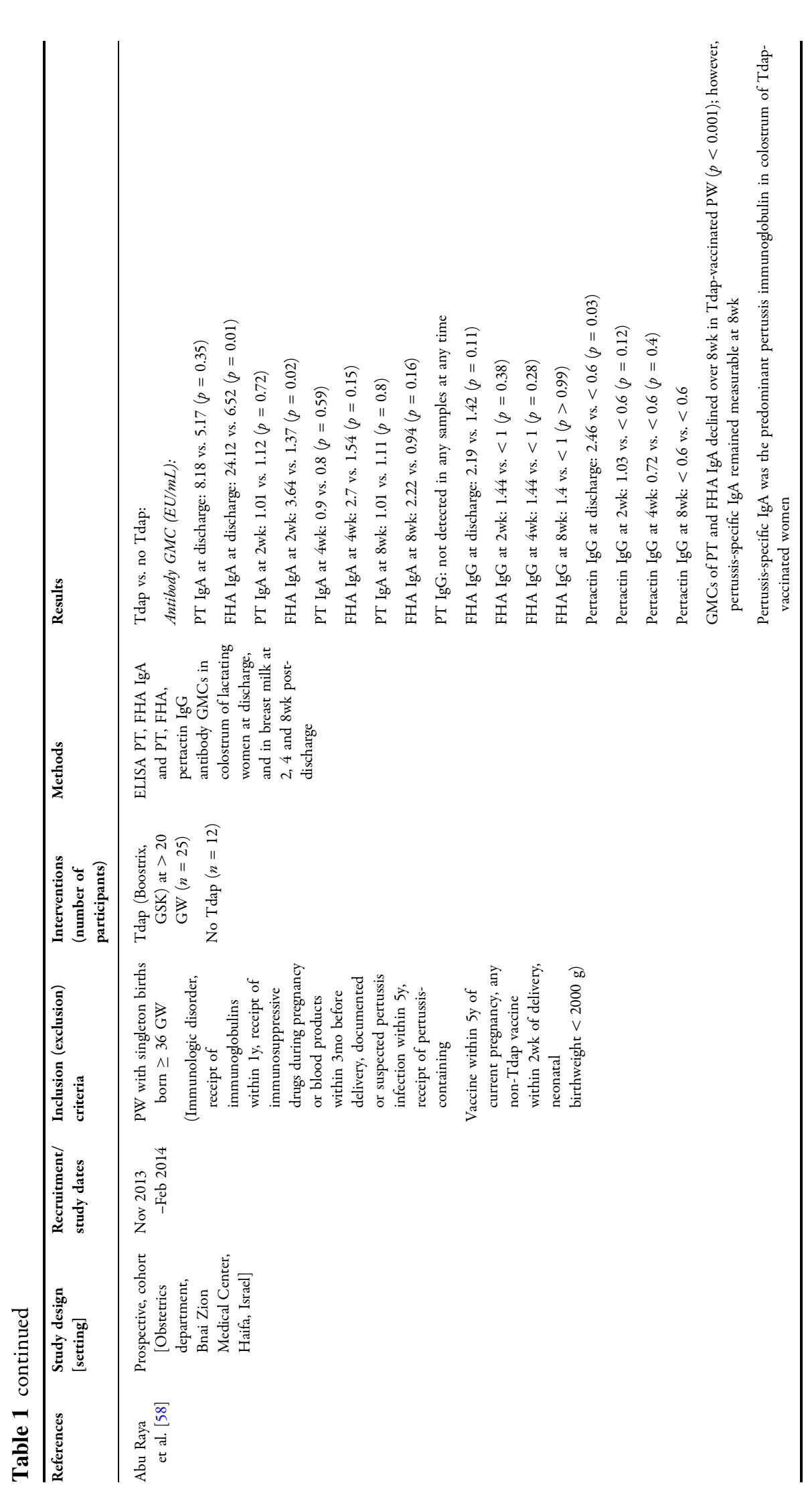




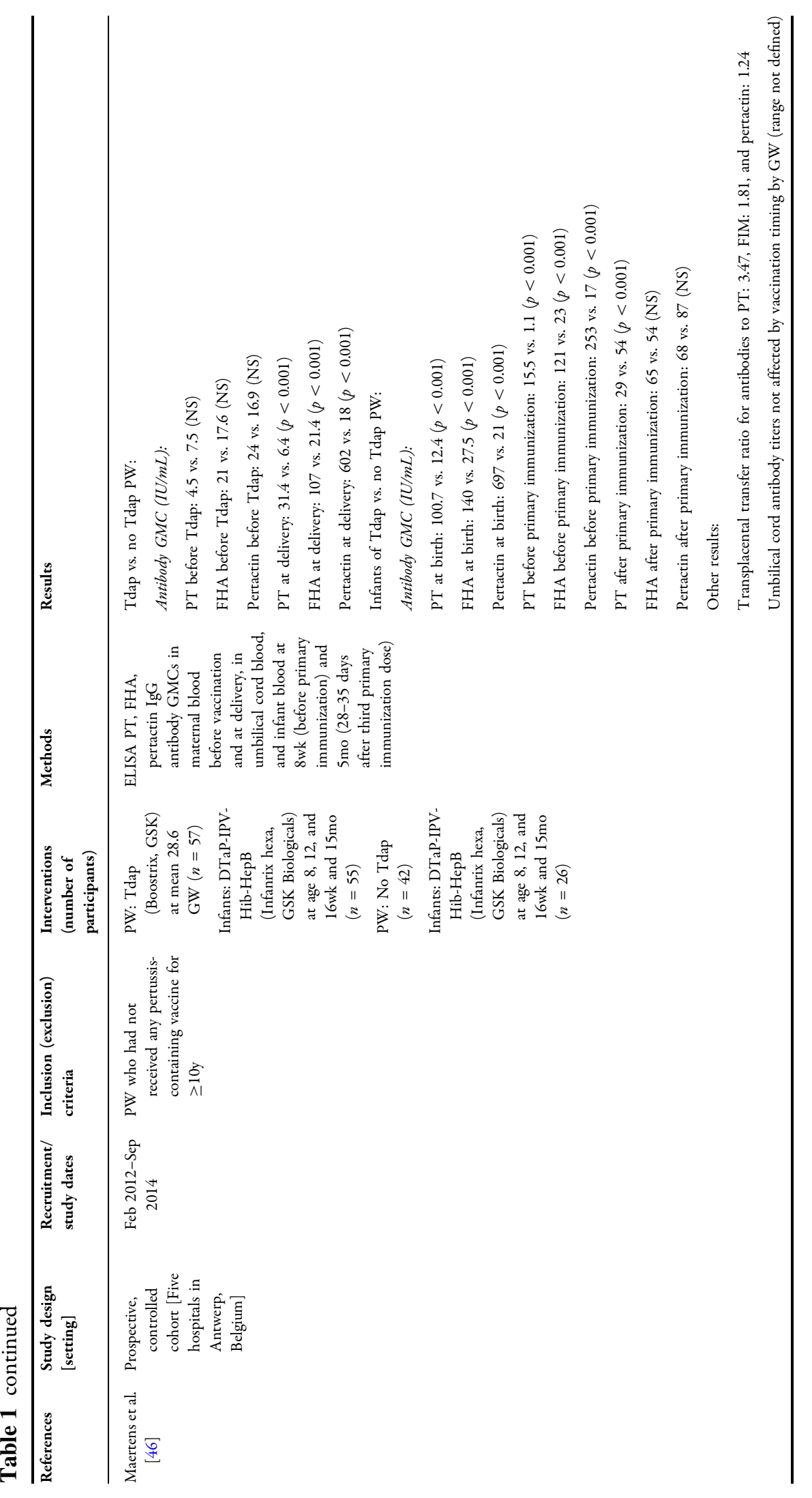




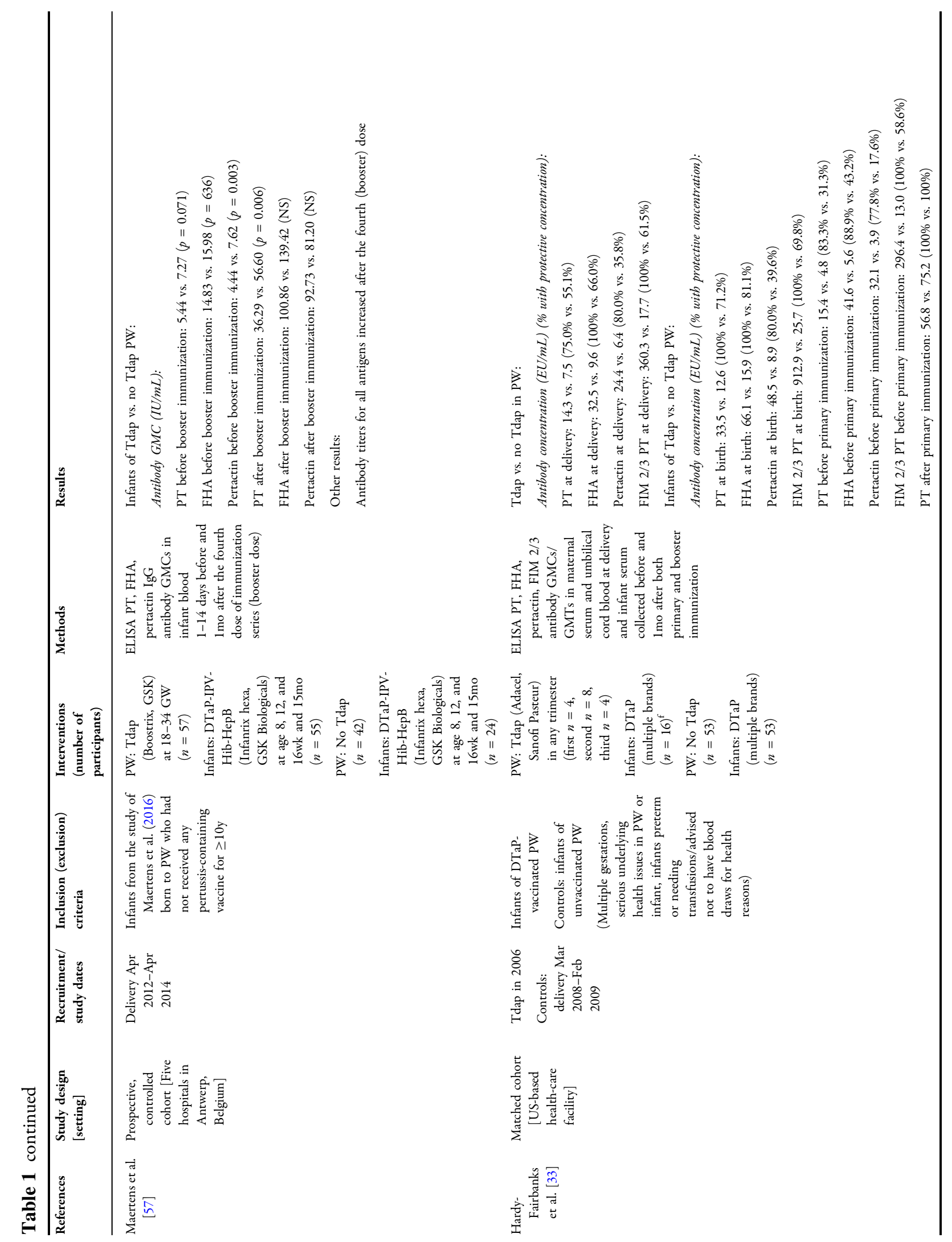




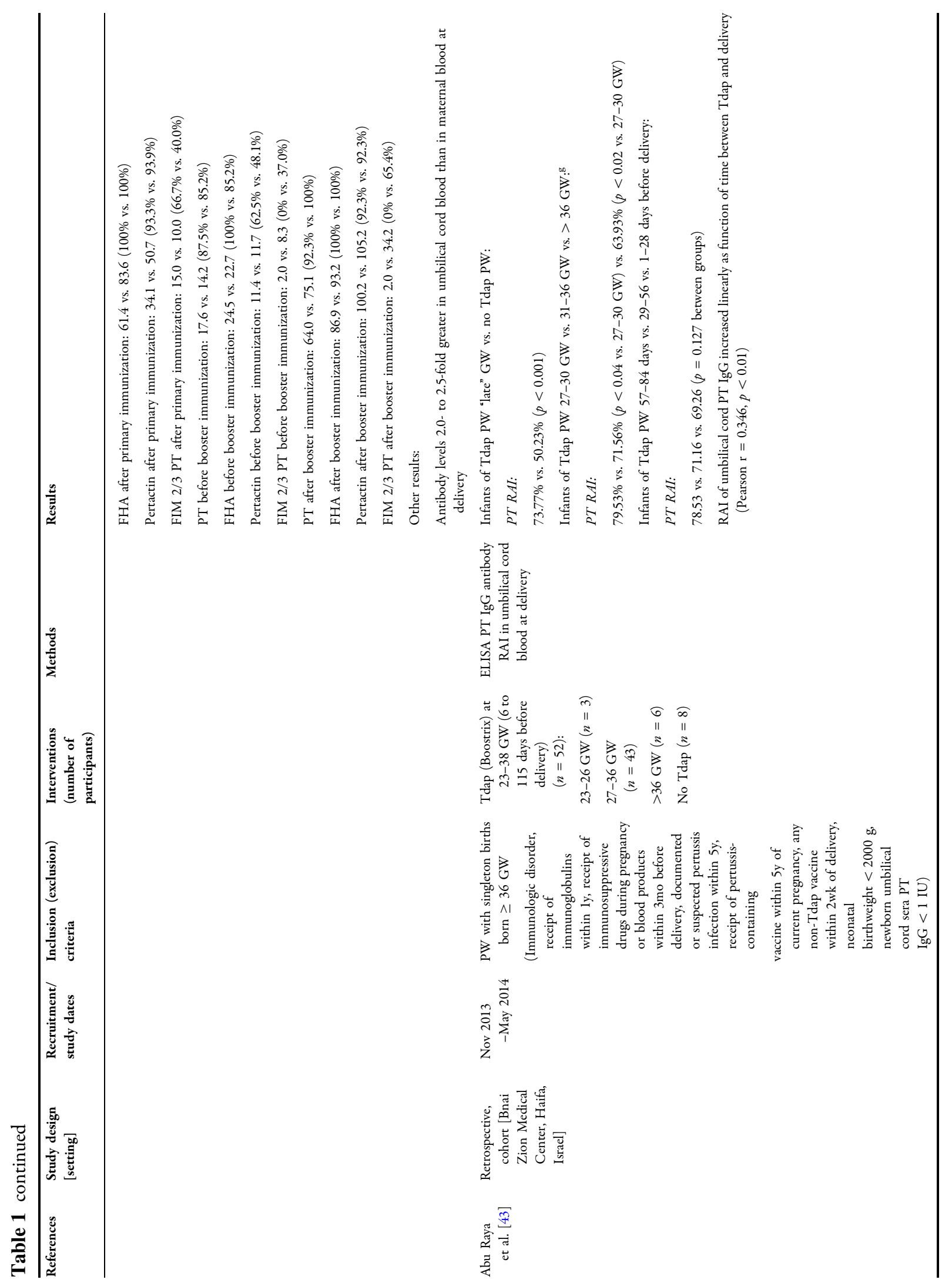




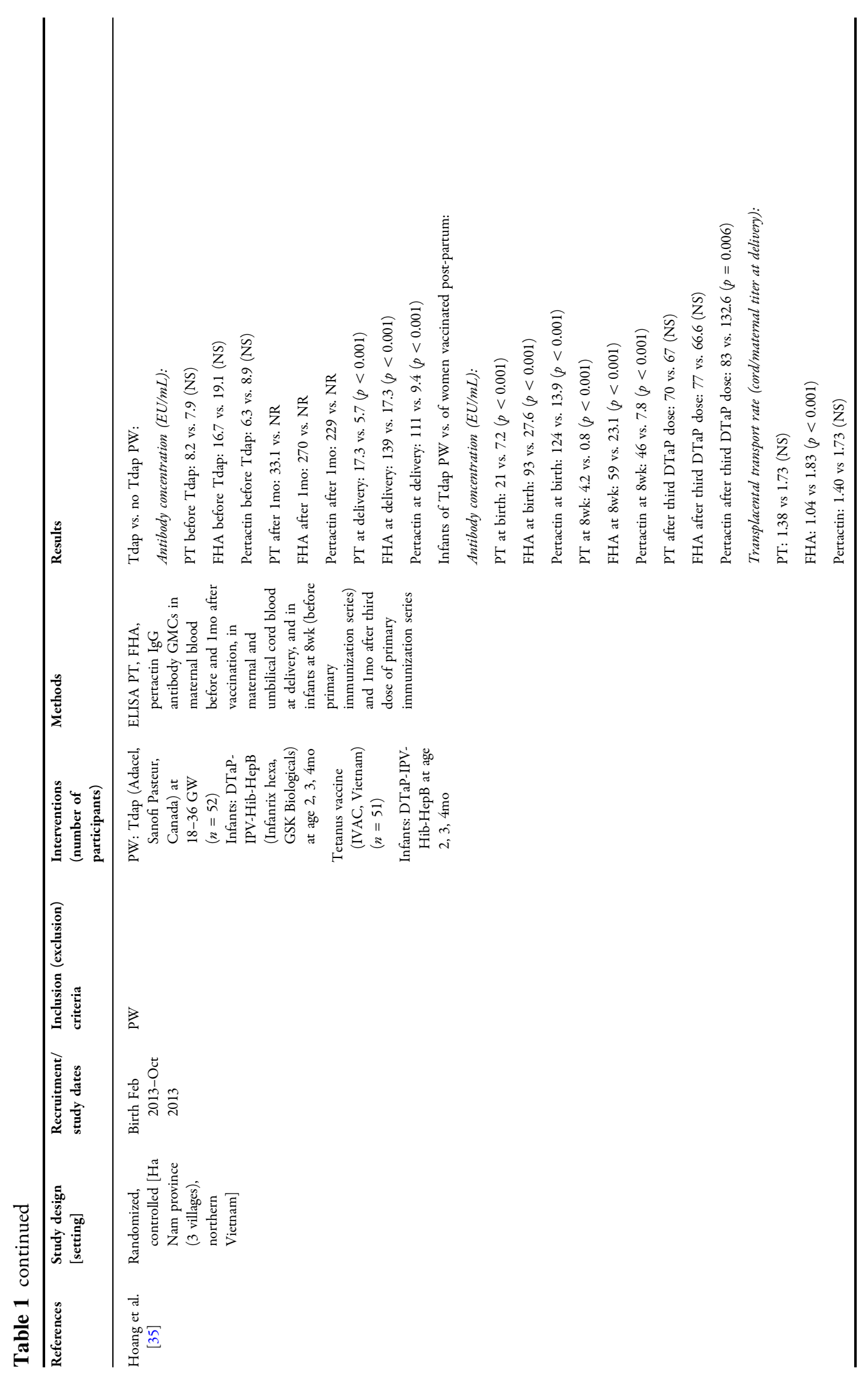




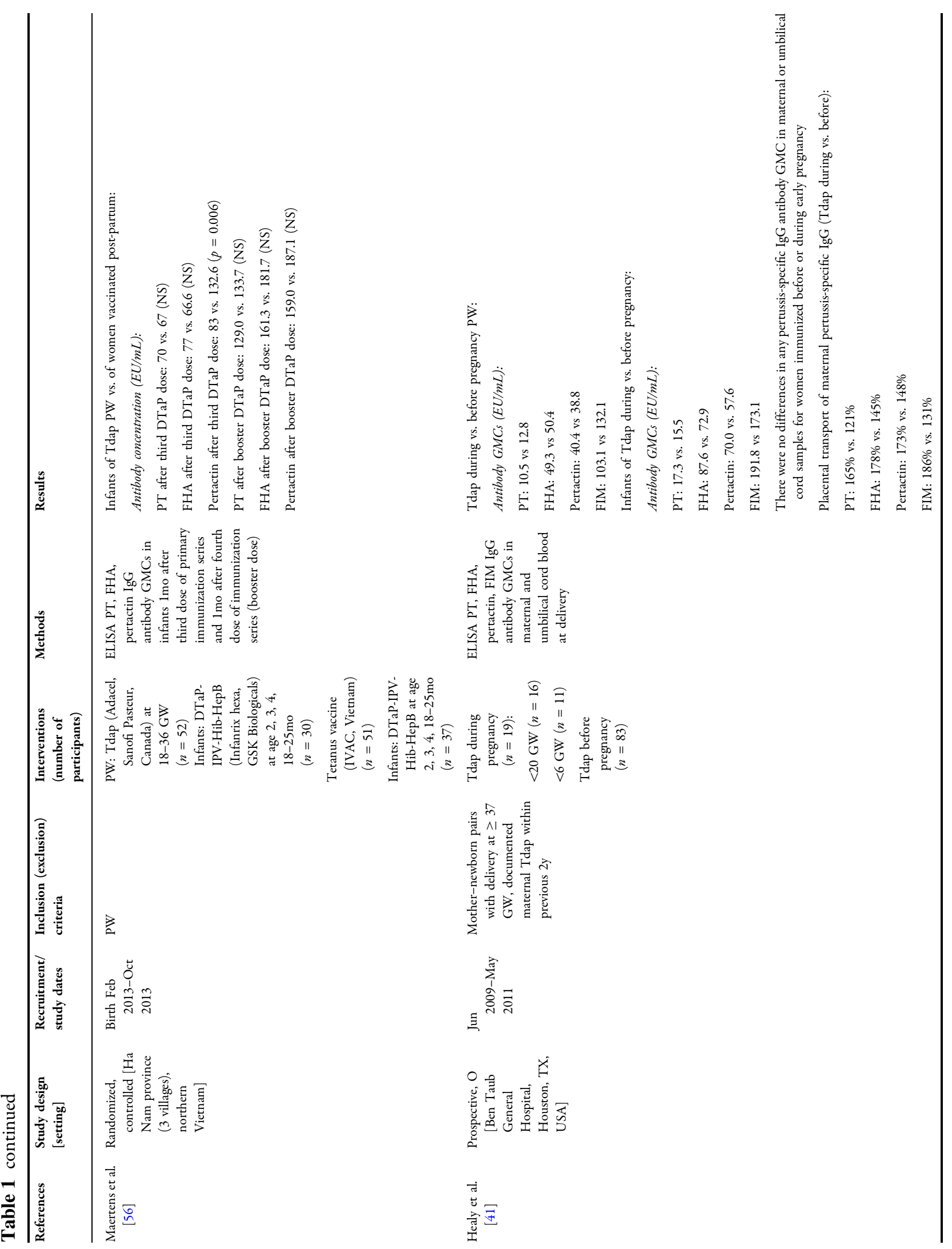




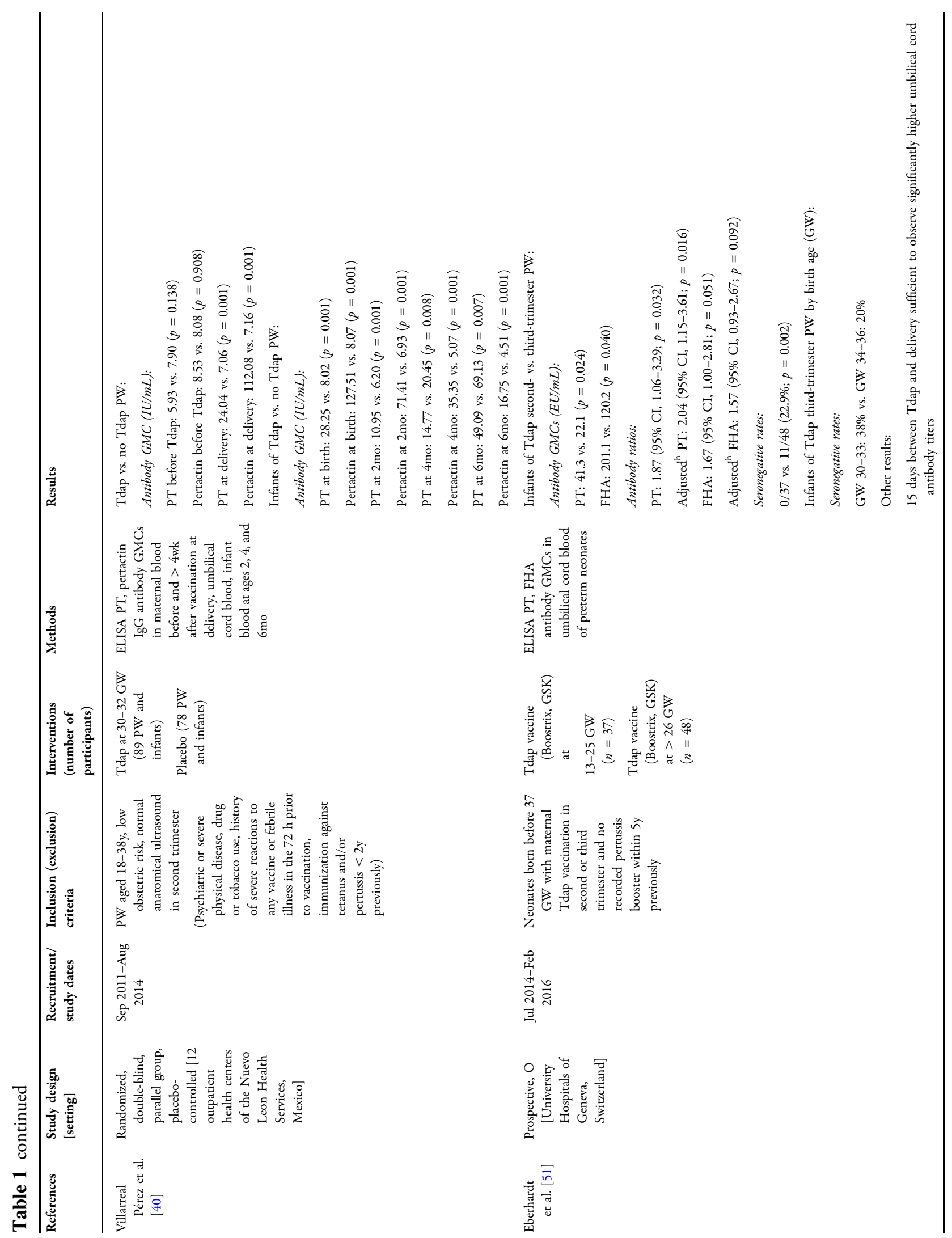




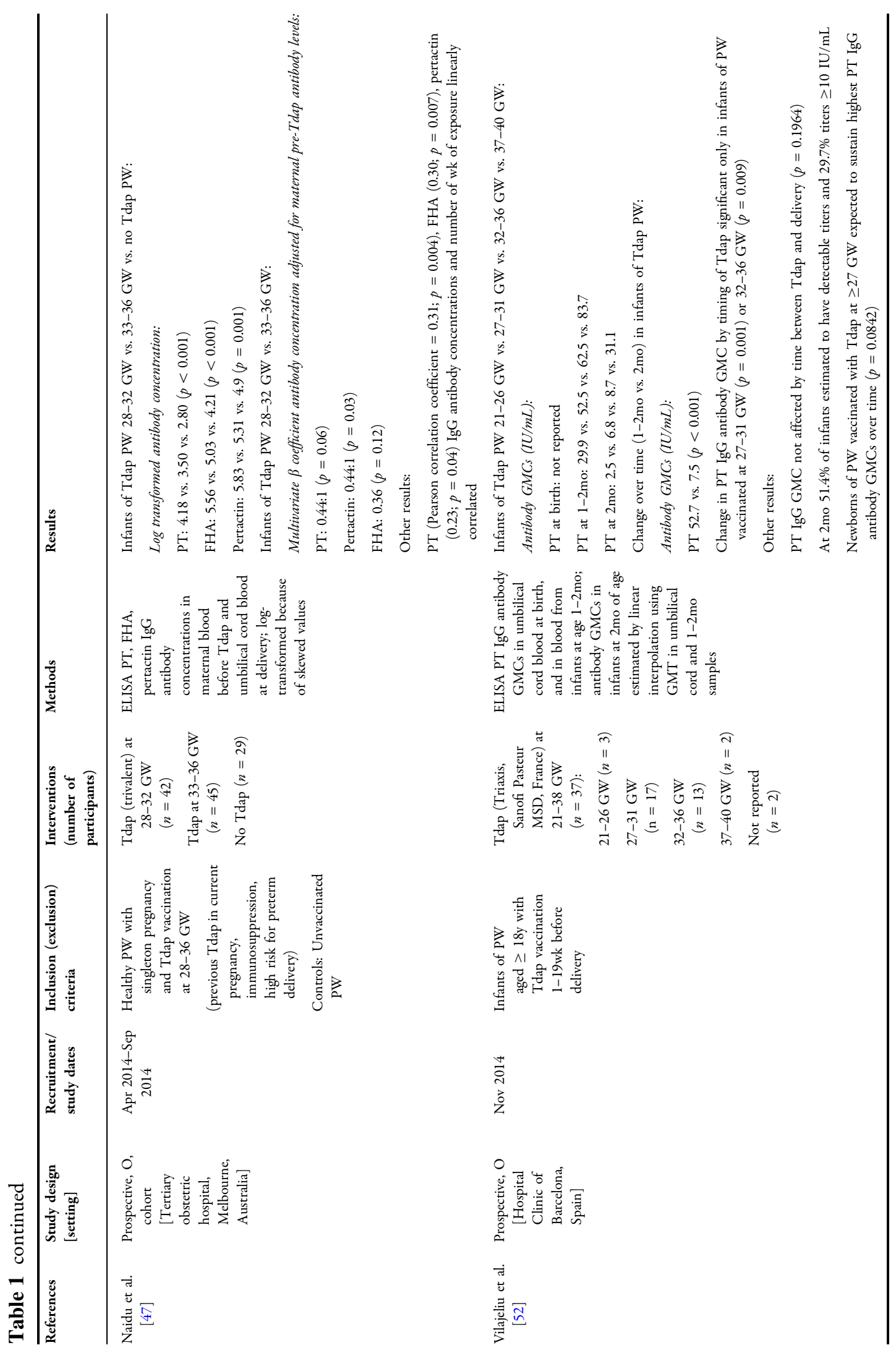




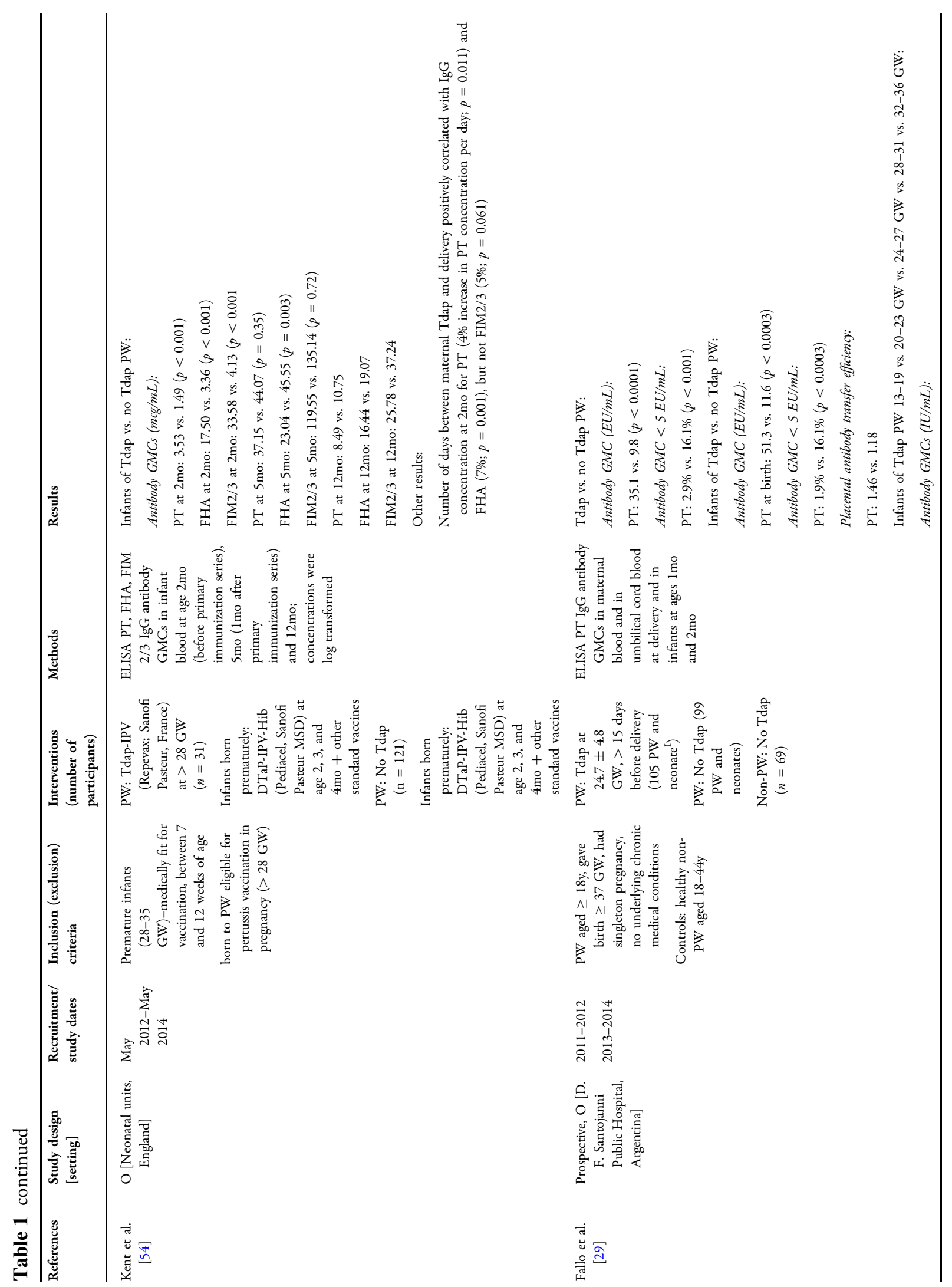




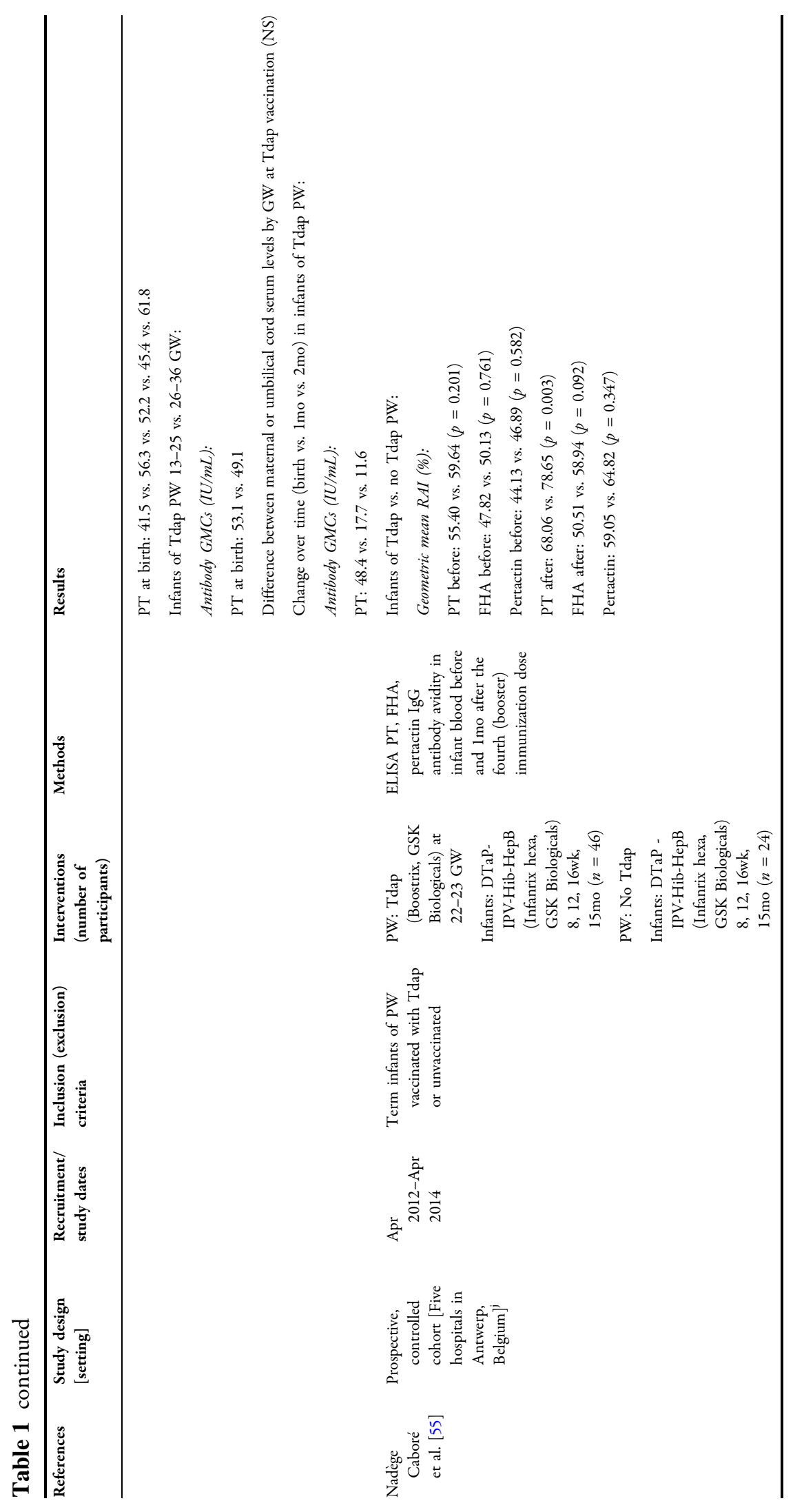




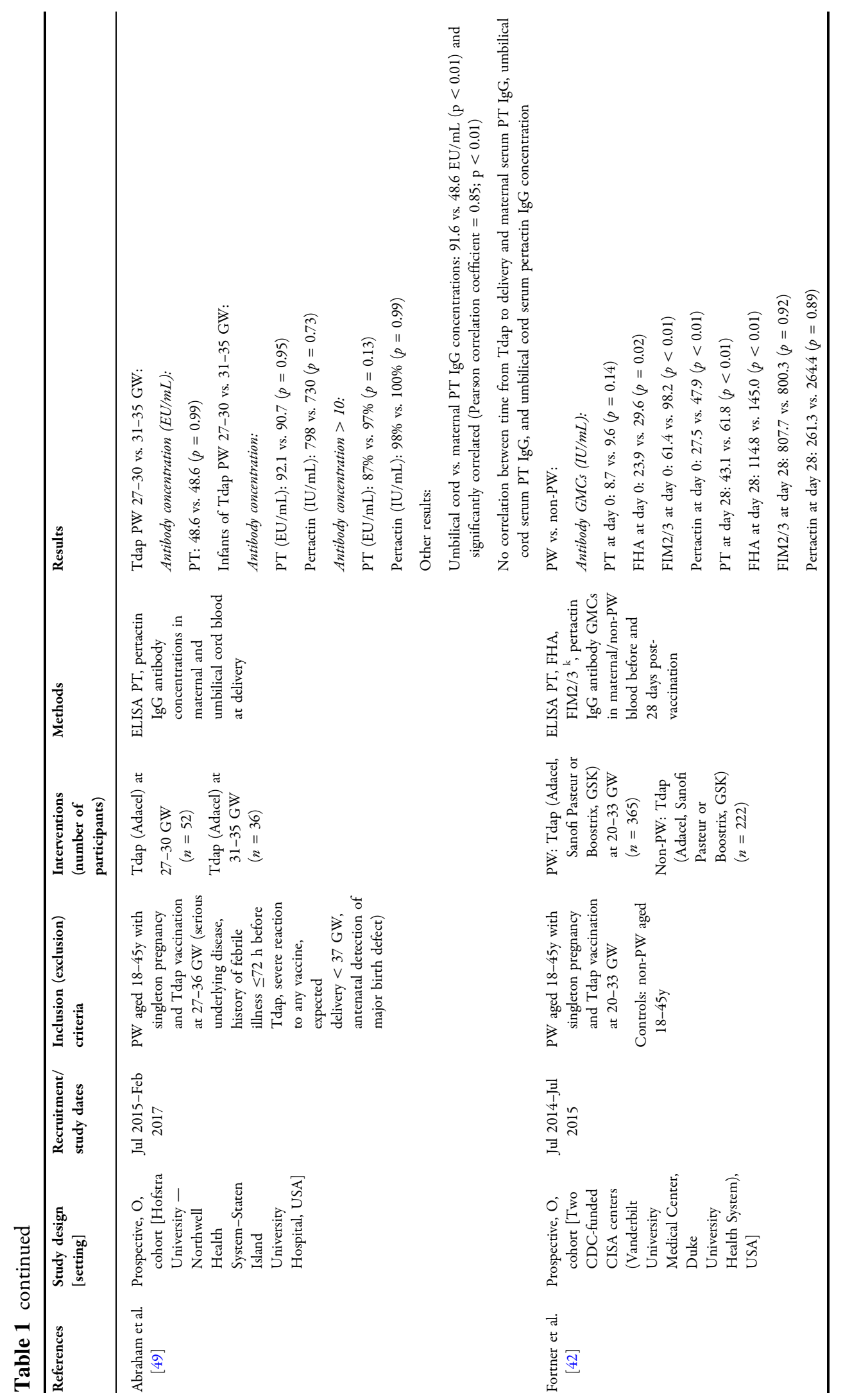




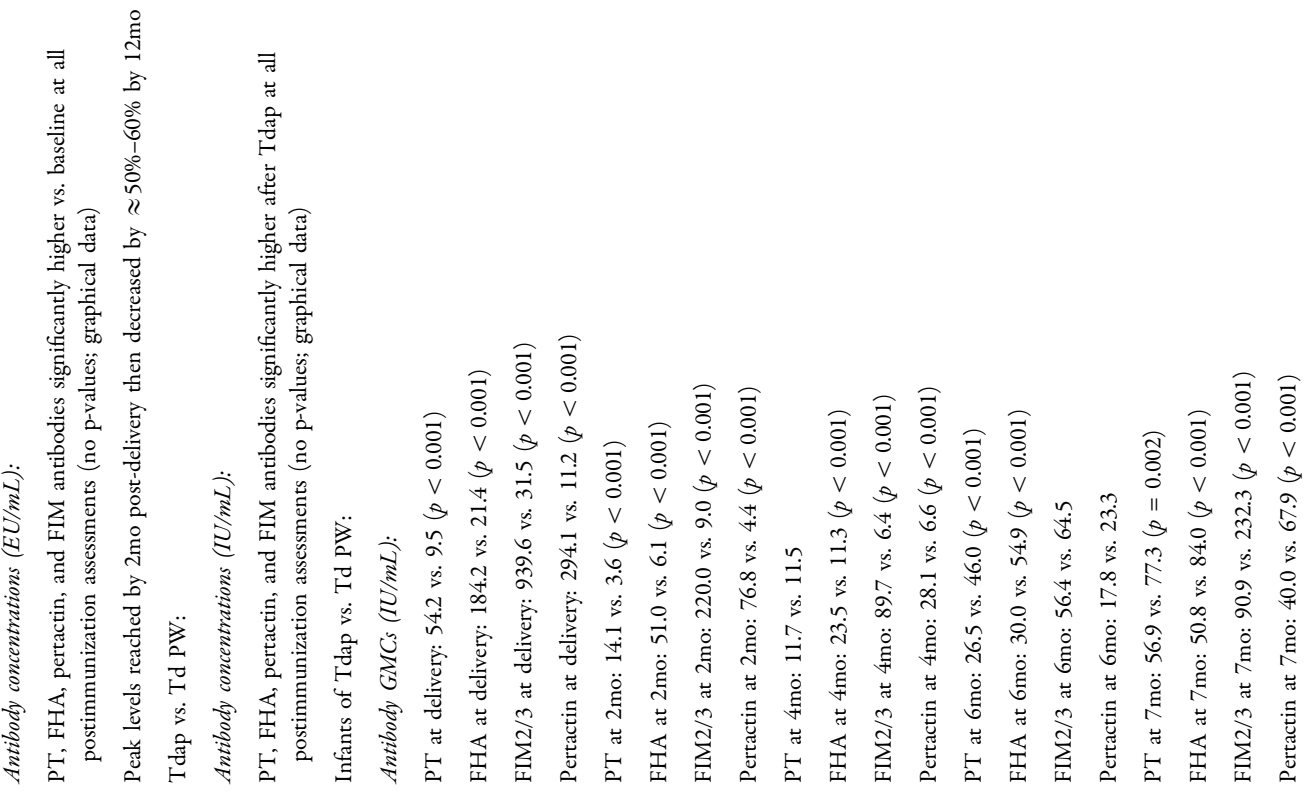

๕

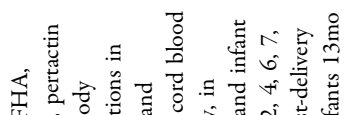

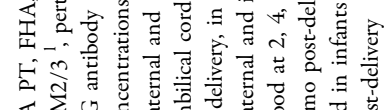

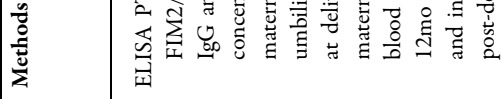

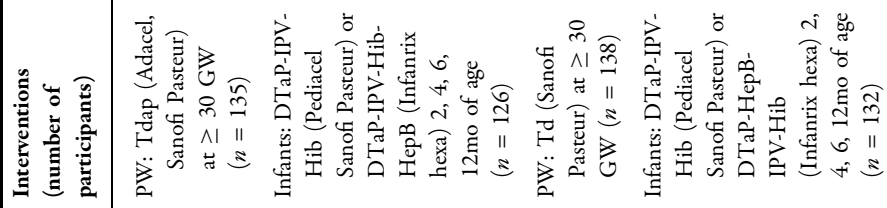

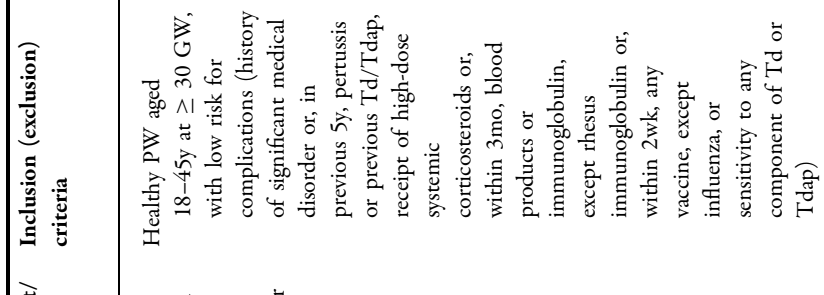

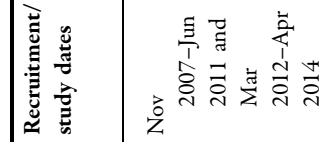

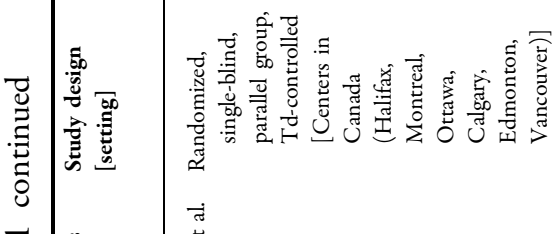

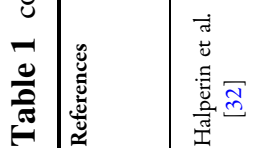




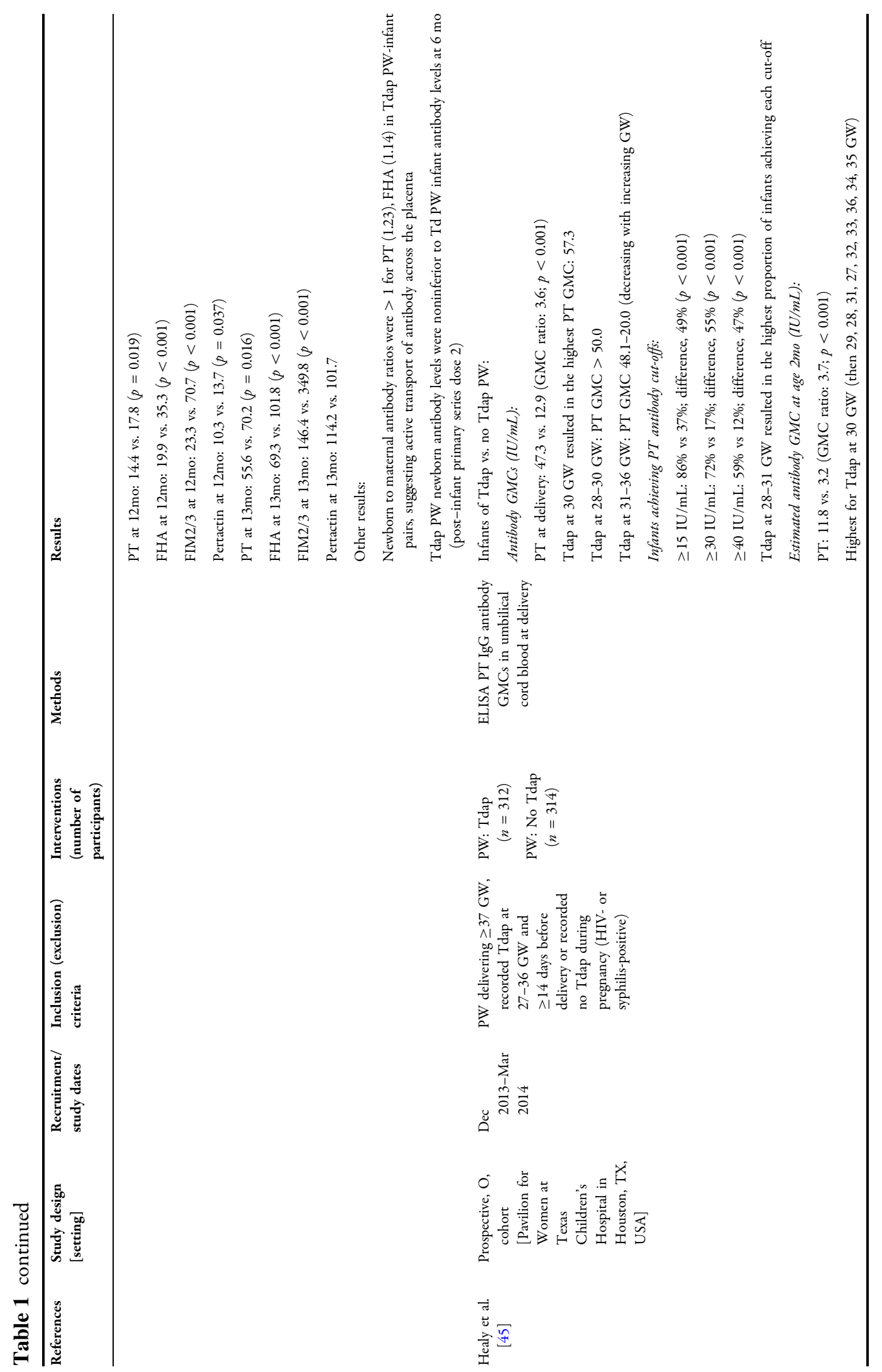




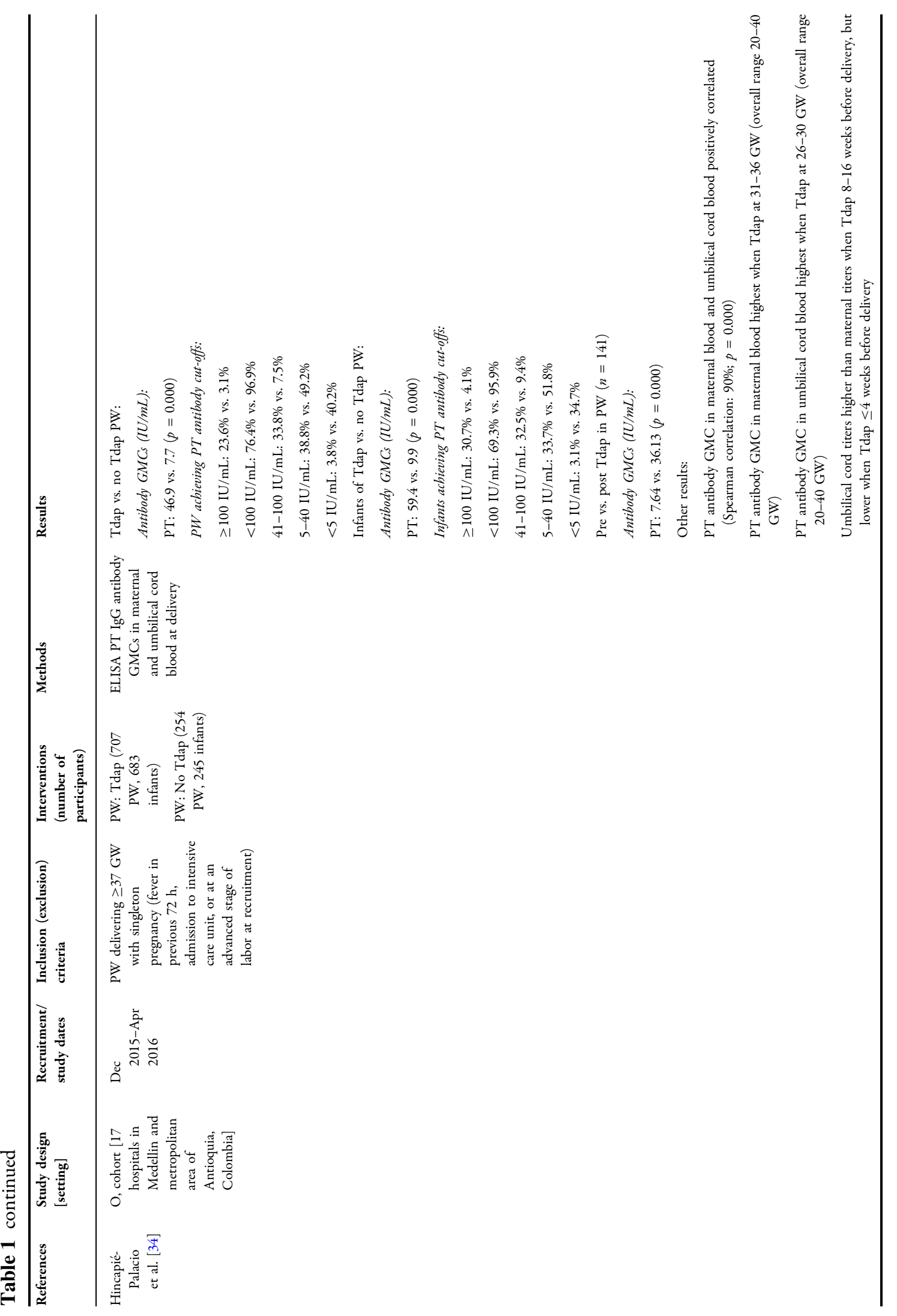




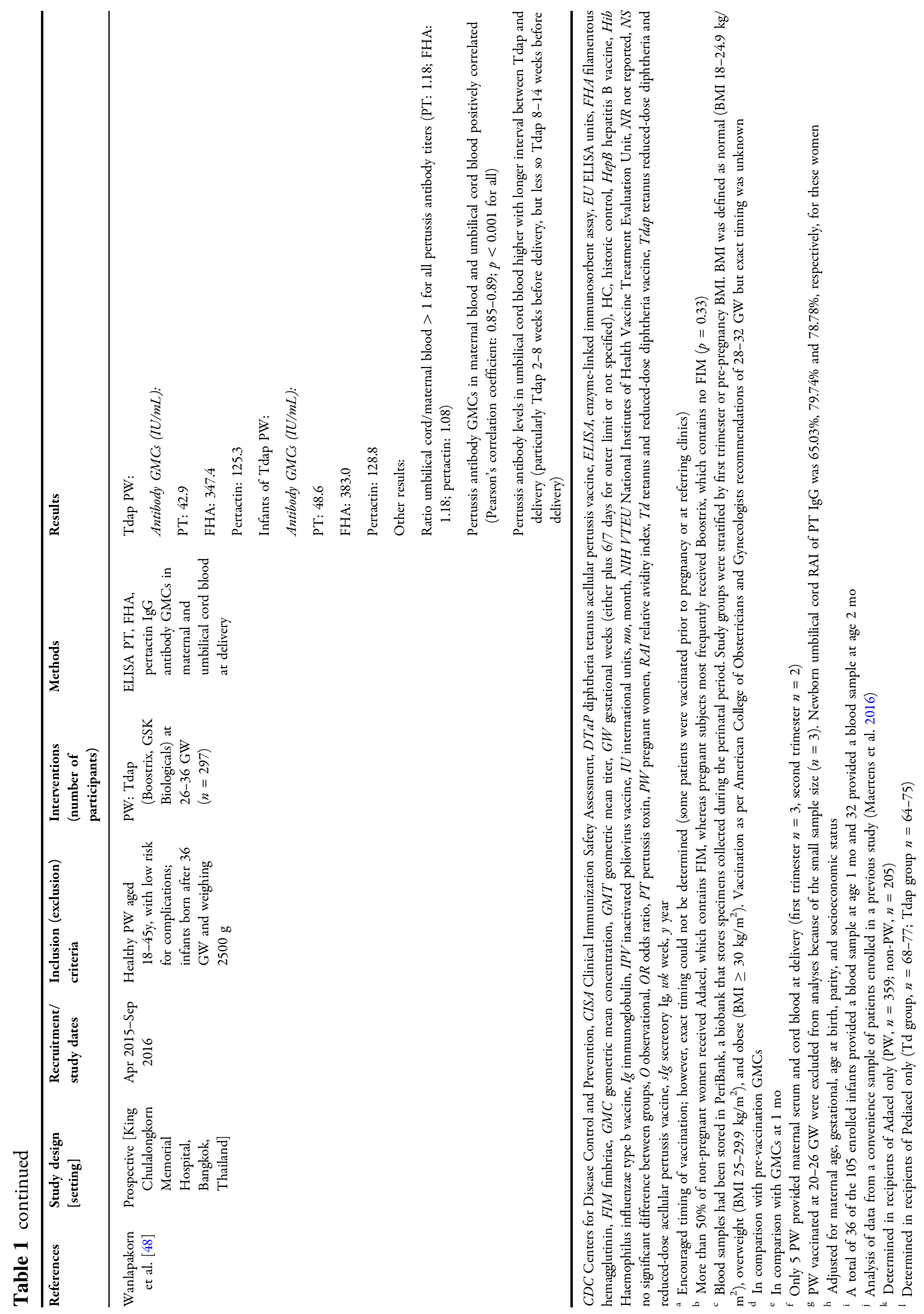




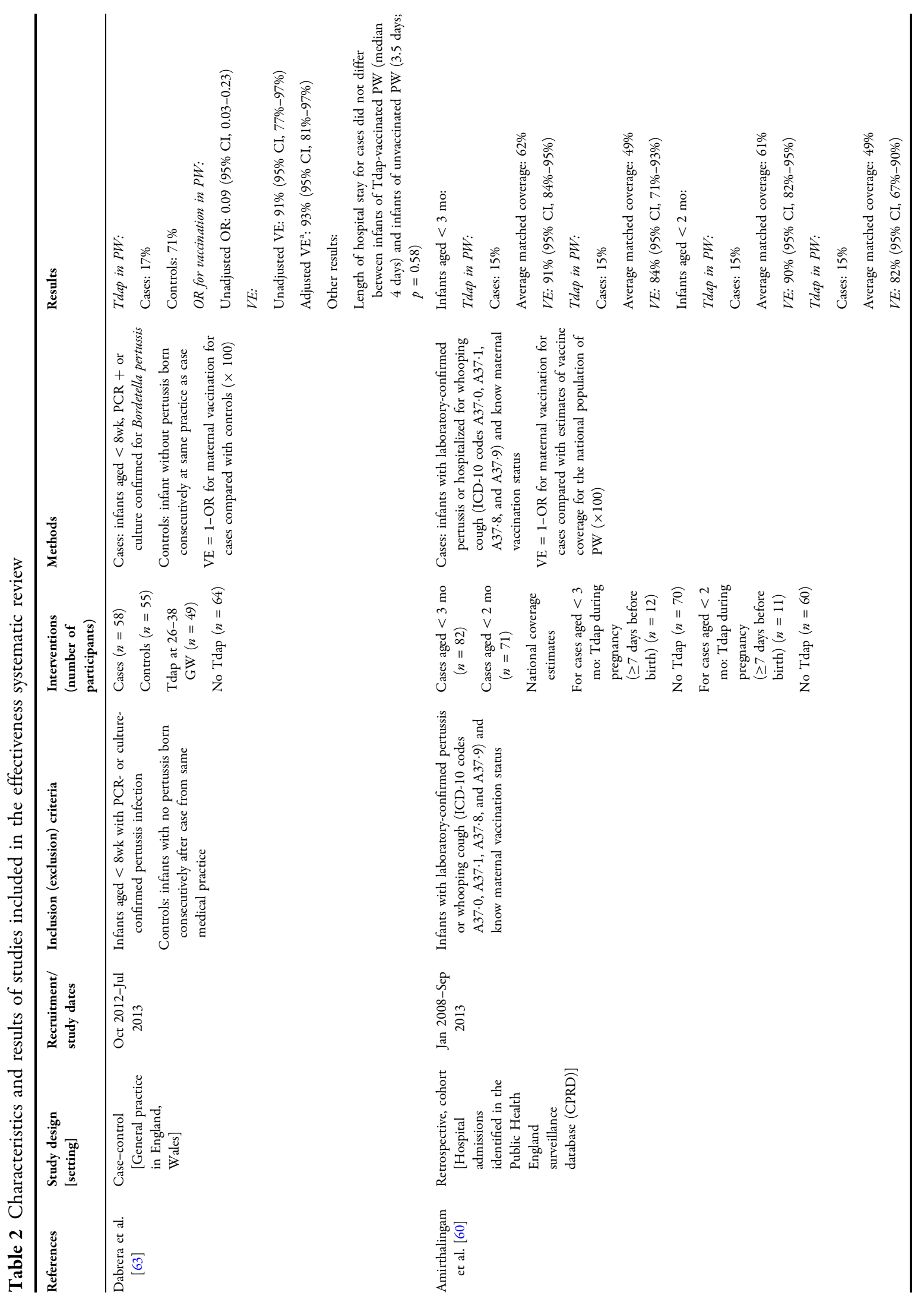




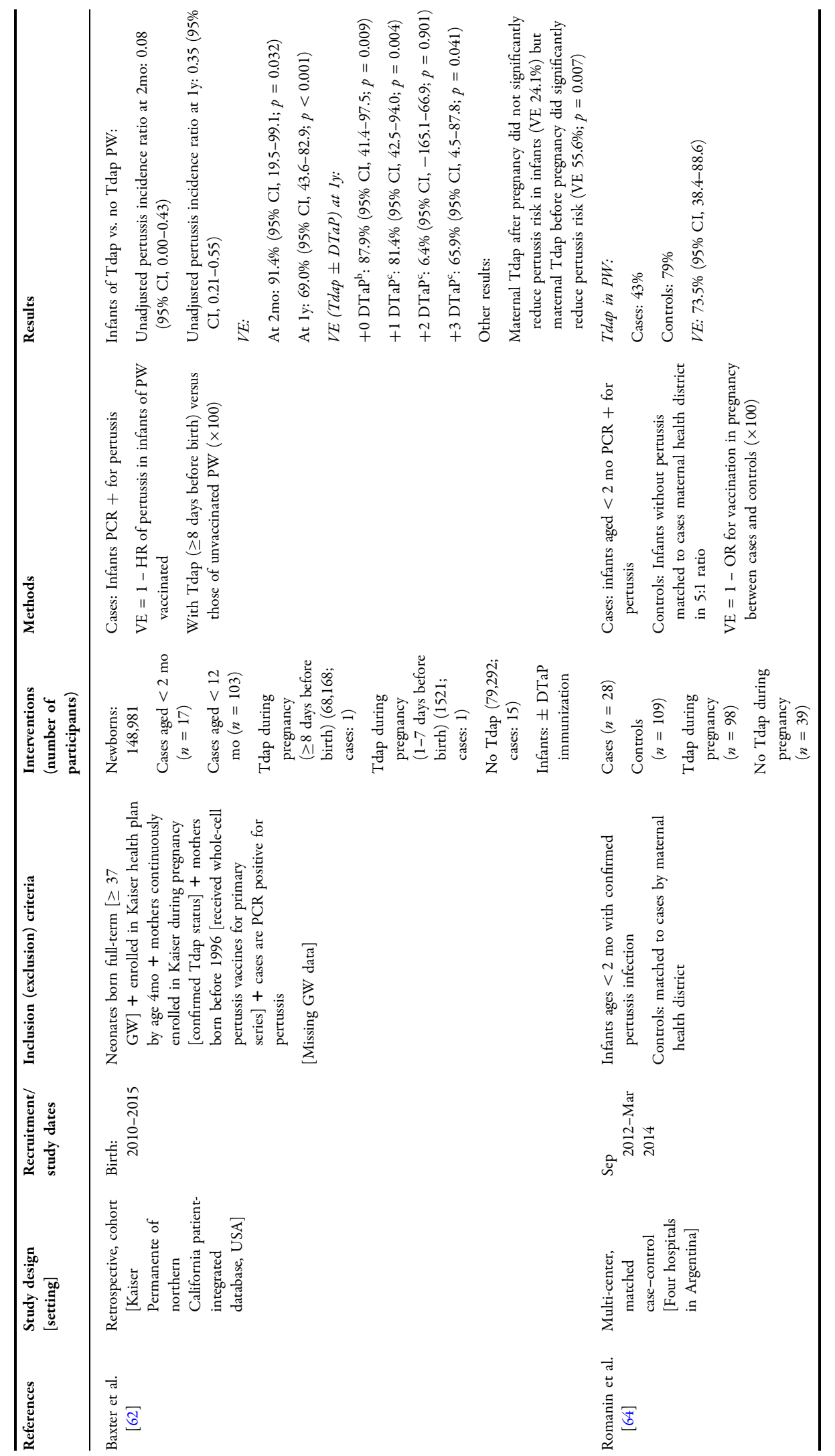




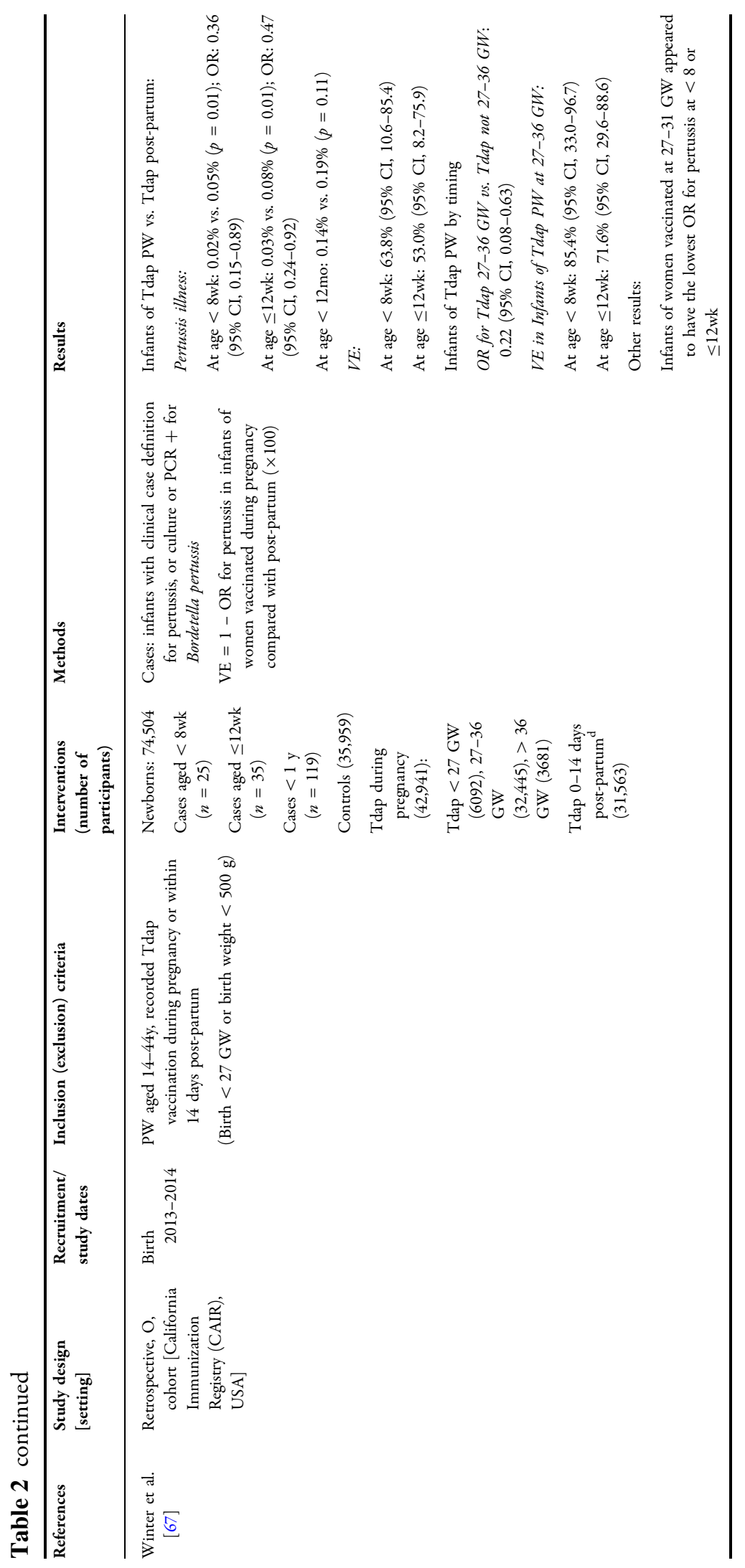


\begin{tabular}{ll}
528 & Infect Dis Ther (2019) 8:499-541 \\
\hline
\end{tabular}

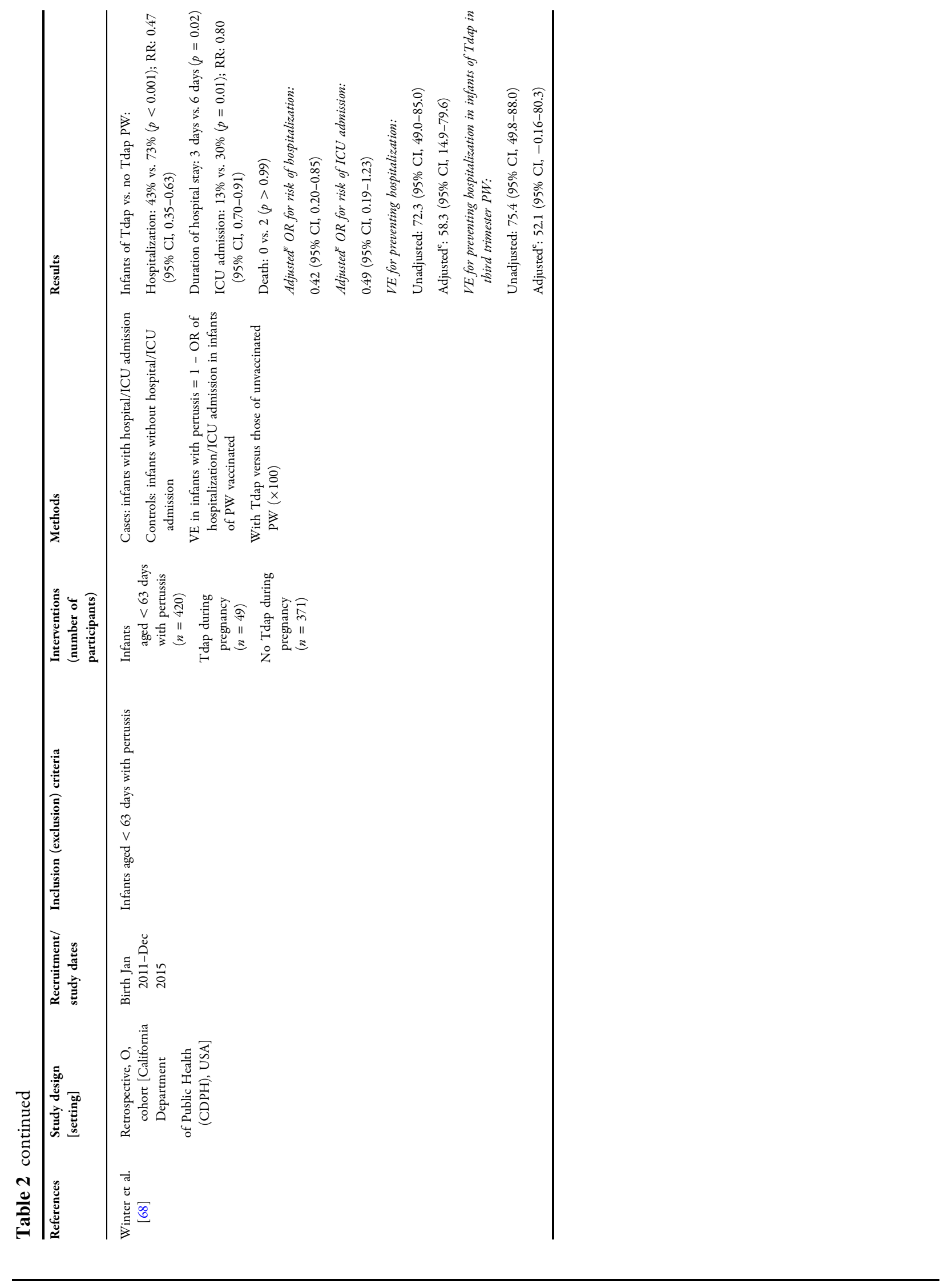

$\triangle$ Adis 


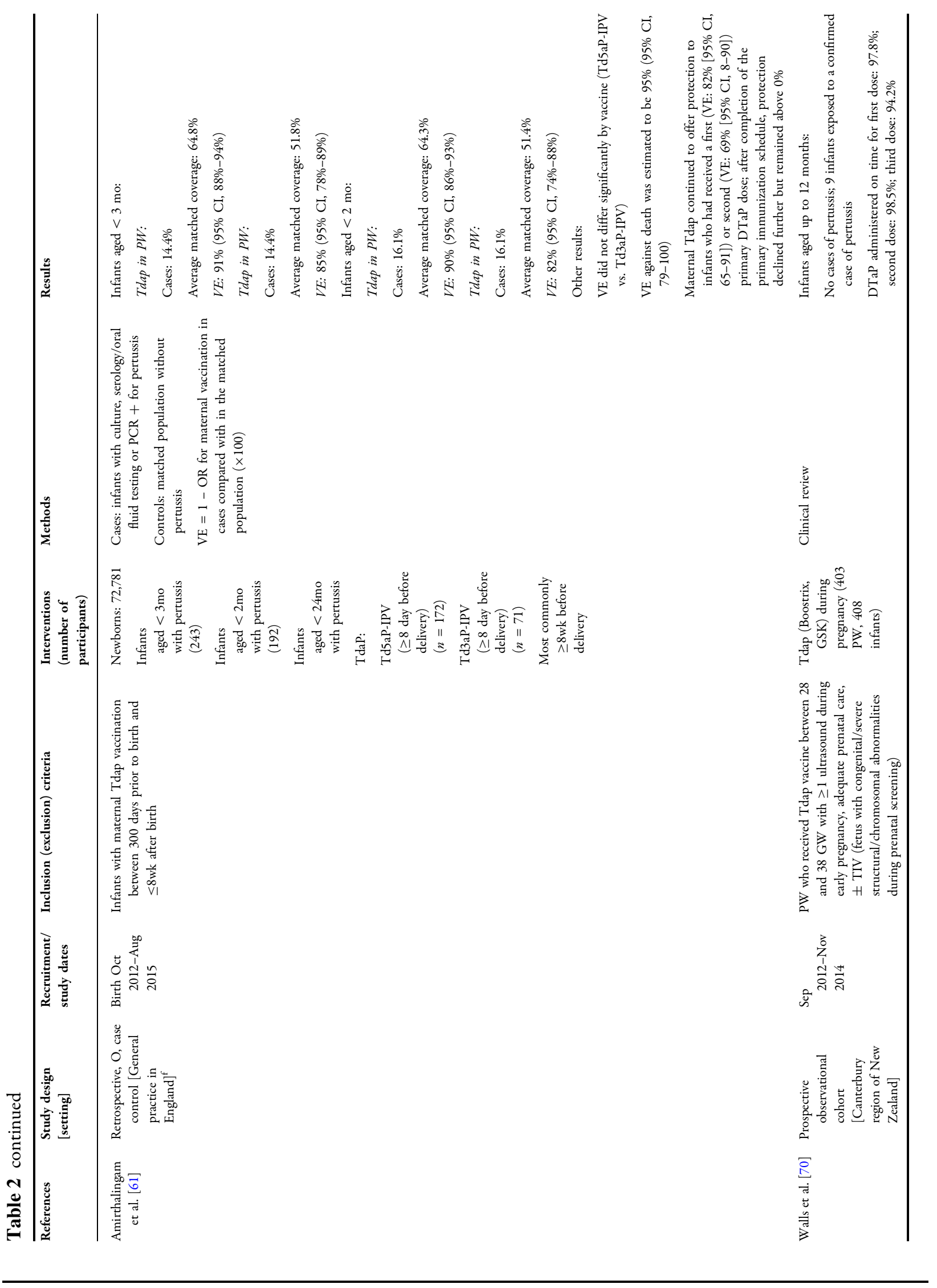




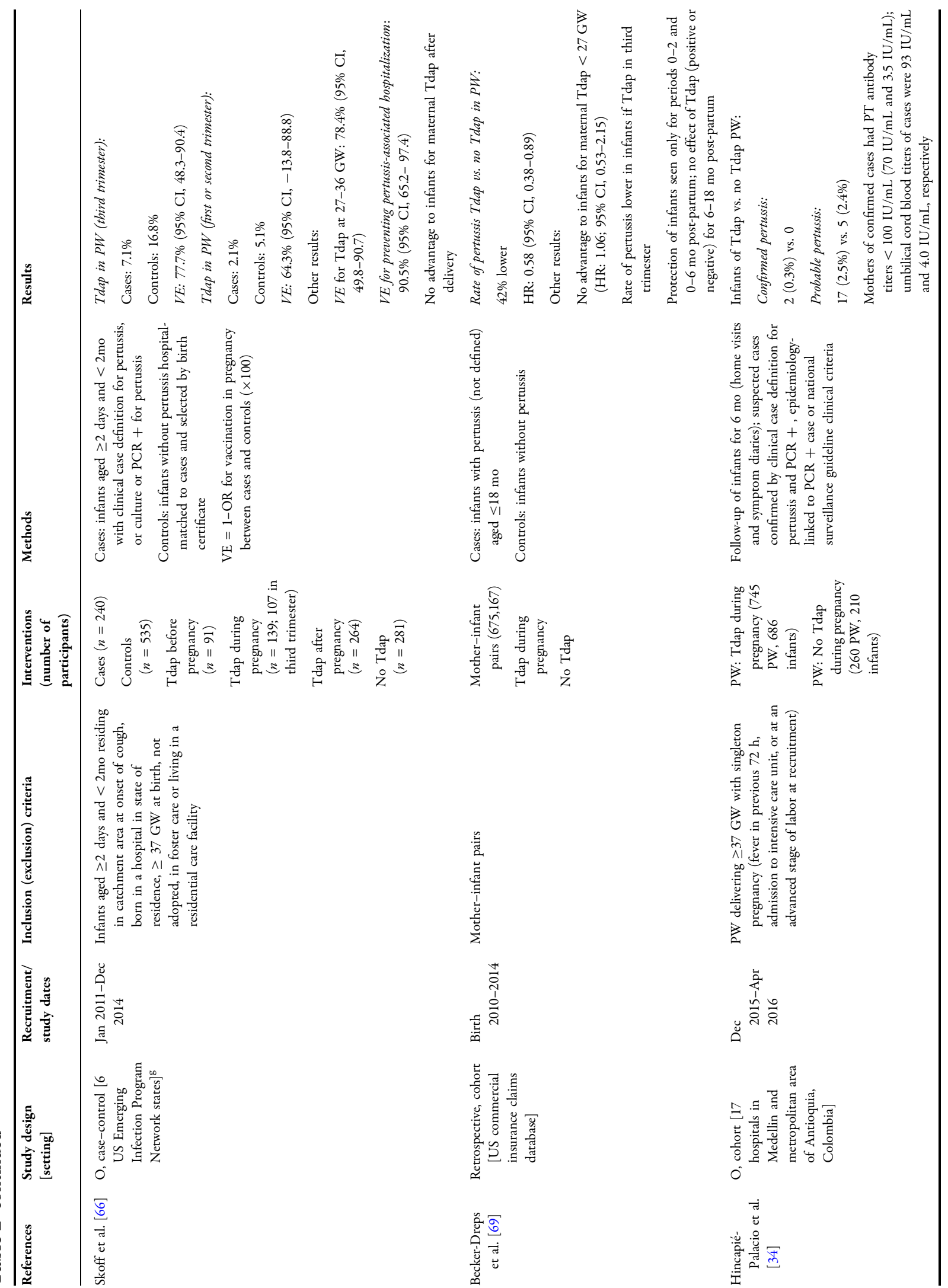




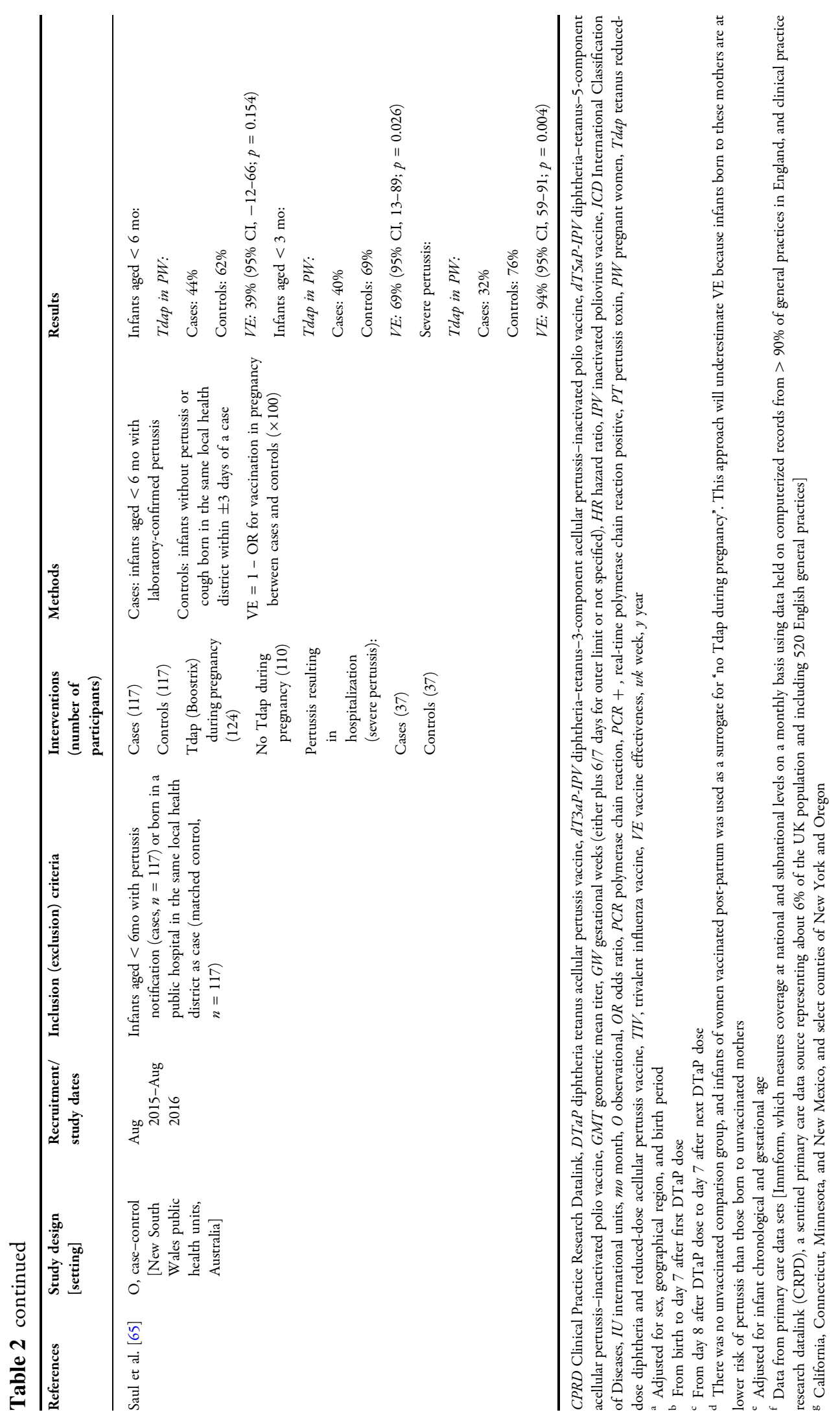




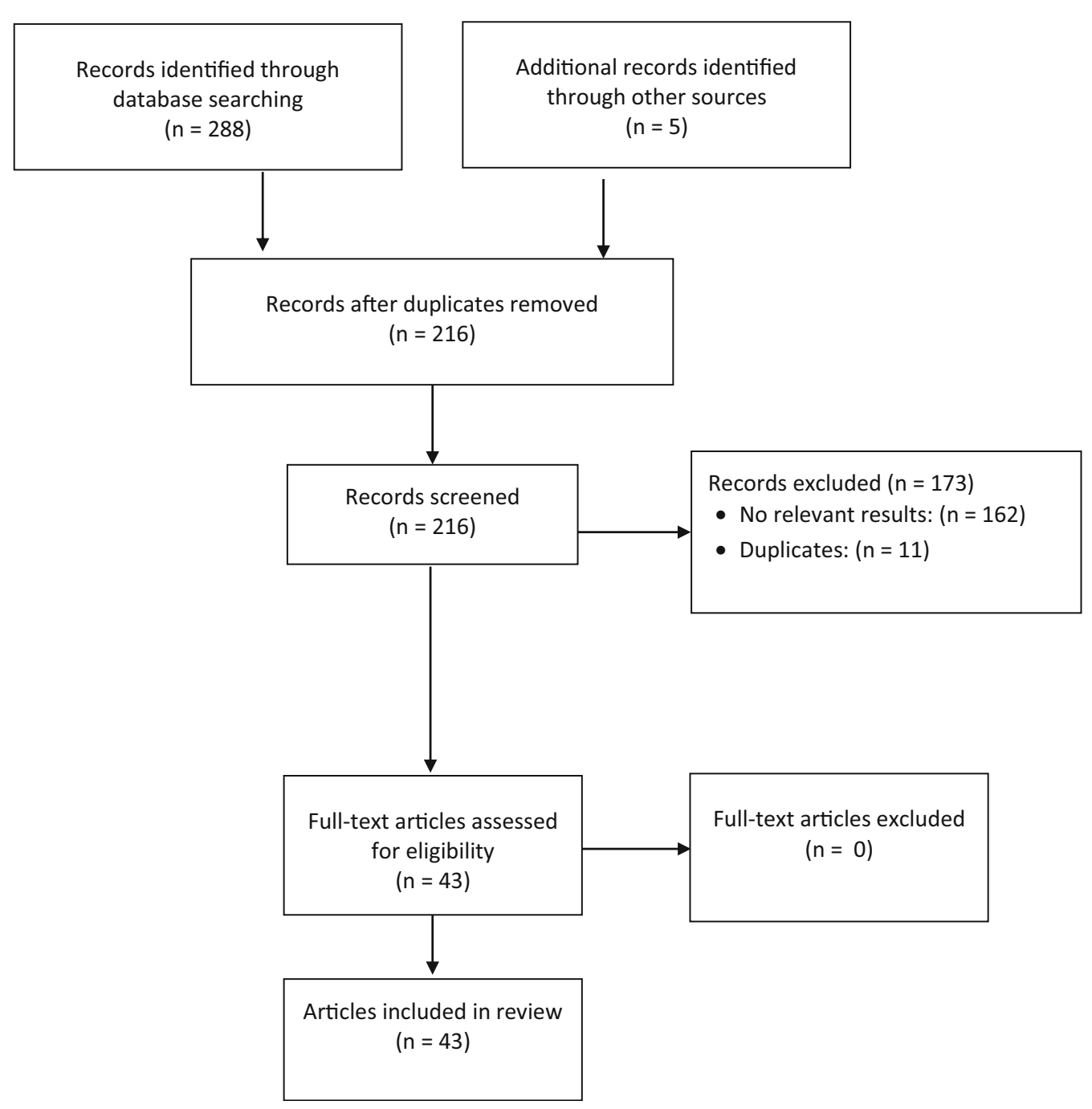

Fig. 1 PRISMA diagram of results of search strategy

fimbriae (FIM)], in the Tdap-vaccinated group versus Tdap unvaccinated control groups across studies (Table 1). The higher antibody levels in Tdap-vaccinated women persisted through to delivery, and for up to $12-15$ months post-delivery in some follow-up studies [28, 32].

Antibody responses to Tdap vaccination during pregnancy appear similar to those in non-pregnant women and women immunized post-partum [36, 38, 41]. However, although antibody GMTs increased for all antigens postvaccination in both pregnant and non-pregnant women in one study [42], those against PT and FHA were significantly higher in non-pregnant women. The difference in the latter study may have simply reflected that a higher portion of non-pregnant women had received prior Tdap vaccination ( $65 \%$ vs. $53 \%)$.

\section{Infant Serological Outcomes-Transfer of Antibodies}

Newborn infants of mothers who received Tdap during pregnancy consistently had higher antipertussis antibody levels (antibodies to PT, FHA, pertactin and FIM) versus newborns of nonvaccinated mothers $(p \leq 0.002$ across antibodies and studies; Table 1) [27, 29, 32, 34, 35, $38,40,43-47]$. Estimates of cord to maternal blood antibody ratios from studies assessing transplacental antibody transfer ranged from 
1.03 to 3.47 across the four pertussis vaccine antigens [27, 29, 32, 33, 35, 38, 39, 41, 46, 48]. A linear positive correlation was found between maternal and newborn's antibody levels $[29,34,38,44,49]$. This correlation may be important for defining the optimal vaccination window during pregnancy since timing may impact the level of antibodies in the mother pre-partum and consequently the level of antibodies transferred to the infant at birth.

The relationship between the timing of Tdap vaccination during pregnancy has been explored, both with respect to gestational week and interval before delivery, and anti-pertussis antibody GMCs in the newborn. A study involving 105 pregnant women found no differences in umbilical cord serum levels and gestational week of Tdap vaccination (13-19 vs. 20-23 vs. $24-27$ vs. $28-31$ vs. $32-36$ weeks) [29]. However, in a larger cohort (335 pregnant women), Eberhardt et al. [50] showed that vaccinating women in the second versus the third trimester resulted in higher umbilical cord antibody levels $(p<0.001)$ and infant seropositivity rates (PT antibody concentration $>30$ $\mathrm{EU} / \mathrm{mL}$ at birth; $p<0.001)$. When second trimester vaccination was compared with vaccination at 26-36 weeks gestation, superiority was again shown for second trimester vaccination [50].

Transplacental transfer of anti-pertussis antibodies to the newborns appears more efficient with maternal Tdap immunization earlier in the third trimester than later $[27,34,43,45,47]$. The transfer ratio was also found to be positively impacted by earlier Tdap vaccination [27, 34, 45]; for example, transfer ratio estimates fell from 1.12-1.45 across antigens in the group vaccinated between 27-30 weeks to $0.97-0.98$ in the group vaccinated after 36 weeks of gestation [27]. The optimal time for vaccination was suggested as 30 gestational weeks, based on umbilical cord antibody GMCs and estimated GMCs in infants aged 2 months [45]. However, pertussis antibody concentrations in umbilical cord blood at delivery did not differ significantly between groups with maternal vaccination at 27-30 gestational weeks or at 31-35 gestational weeks in another study [49].
Although umbilical cord pertussis antibody levels were positively correlated with the interval between vaccination and delivery (a period of about 2-13 weeks of vaccine exposure was considered) [47], this correlation does not seem to extend to pertussis immunization very early in pregnancy. Healy et al. found that, although vaccination before pregnancy or early in pregnancy both resulted in increased antibodies at birth, there was no difference $(p \geq 0.45)$ between GMCs of newborns in the two groups [41]. Similarly, cord blood PT and FHA antibody GMCs were similar in infants whose mothers were immunized $<2$ weeks prior to delivery or not immunized, suggesting that vaccination $<2$ weeks prior to delivery was unlikely to effectively protect the newborn [50]. Optimal timing of vaccination, based on umbilical cord antibody GMCs, was determined to be 30-120 days before delivery (range considered $<15->150$ days) [50], although 15 days was considered sufficient to significantly elevate umbilical cord antibody GMCs in a preterm population [51]. Other studies have suggest that vaccination $\geq 8$ weeks before delivery may maximize antibody levels to pertussis antigens in umbilical cord blood [27, 34, 48], with the optimal timing being 57-84 days before delivery (range considered 1-84 days) [27]. Of note, the timing of vaccination may not only affect the quantity of antibodies transferred but also their functionality, since the relative avidity of antibodies in cord blood increased linearly with time between Tdap vaccination and delivery [43]. In contrast, two studies found no correlation between time from Tdap vaccination to delivery (range 3-21 weeks and about 2-14 weeks, respectively) and pertussis antibody concentrations in umbilical cord blood at delivery $[39,49]$.

Transplacentally acquired antibodies in early infancy decay over time up until receipt of the first dose of the primary pertussis vaccination series [29, 32, 33, 35, 38-40, 46, 52]. Two studies quantified this decay at $58-76 \%$ across the pertussis-specific antibodies [29, 38]. Nonetheless, the anti-pertussis antibody levels remained higher through the period before the first pertussis vaccine (generally 8 weeks), with 3.2-24.4-fold higher antibody GMCs persisting 
in infants of Tdap-vaccinated versus non-vaccinated mothers [32, 33, 35, 38, 40, 45, 46]. Using previously estimated half-life values for maternally acquired antibodies in newborns to model the persistence of anti-pertussis antibodies, it was determined that transferred maternal antibodies would remain detectable at age 2 months in $51-89 \%$ of infants born to mothers who received Tdap during pregnancy $[39,41,52,53]$. These findings suggest that maternal pertussis immunization could bridge the susceptibility gap until the infant receives the first dose of pertussis vaccine.

\section{Infant Immune Responses-Interference with Primary Vaccination}

There is a theoretical risk that transplacentally acquired maternal antibodies may blunt the immune responses and thus the protection elicited by the primary diphtheria tetanus acellular pertussis vaccine (DTaP) immunization series. The available studies on the immunological effect found blunting of antibodies to at least one pertussis antigen following all or part of the primary vaccination series among infants whose mothers received pertussis immunization during pregnancy compared with infants of mothers who did not (Table 1) $[33,35,38,40,46,54]$. Whether the blunting effect also persists with the infant booster vaccination is unclear. Some studies suggest that pertussis-specific antibody GMCs either remained lower $[32,55]$ or did not differ notably $[33,38,56] 1$ month after the booster dose in infants of mothers who received Tdap during pregnancy compared with those of mothers who did not. However, all of the latter studies had small sample sizes, which may have precluded detection of significant differences. In one study, FHA and pertactin antibody levels did not differ 1 month after the booster dose between infants of Tdap-vaccinated versus unvaccinated mothers, but PT antibody levels were significantly lower in the infants of vaccinated mothers [57]. Nonetheless, increases in all relevant pertussis antibody GMCs following the primary and/or booster immunization series are observed in the infants of mother immunized during pregnancy $[33,35,46,54,56,57]$.
A prospective cohort study conducted following implementation of the program of immunization of pregnant women in the UK with Tdap-IPV examined the issue of immunological blunting from a broader perspective, exploring the effect on the whole range of vaccines administered to infants in the first few months after birth [53]. Immunological responses to pertussis, diphtheria, tetanus, Haemophilus influenzae type B, pneumococcal, and meningococcal vaccines in infants of vaccinated mothers were compared with historical data from infants vaccinated before program implementation. Although infants born to vaccinated mothers achieved a 2.64-fold increase $(p<0.001)$ in PT antibodies 1 month after completion of the primary immunization program relative to GMCs before the first primary immunization dose, GMCs for antibodies to FHA and FIM were lower than pre-dose GMCs. In addition, response to all three pertussis antigens was blunted by $33-49 \%$ in comparison with the historical data $(p<0.001$ for all). In the absence of a pertussis vaccine booster dose in the UK national immunization program, the study could not identify whether this blunting persisted with the booster in second year of life.

\section{Antibody Transfer in Breast Milk}

Limited data were identified evaluating the potential for antibody transfer through breast milk and it is unclear whether Tdap vaccination during pregnancy meaningfully affects pertussis antibody concentrations in colostrum or breast milk [58, 59]. While, the value of transfer of breast milk immunoglobulin (Ig)A for clinical protection remains unclear, the data are indicative that secretory antibodies generated by the vaccine are also likely to have been transferred in utero to the fetus and contribute to protection. Abu Raya et al. (2014) found that GMCs of IgA to PT were similar in the colostrum and breast milk of Tdap-vaccinated and unvaccinated women at weeks 2,4 and 8 post-partum [58]. However, IgA to FHA was significantly higher in colostrum and breast milk from vaccinated women at 2 weeks. IgA to both pertussis antigens significantly declined over the 8-week study period. In the study by De Schutter et al. 
(2015), women vaccinated during pregnancy $(p=0.012)$ or at, or shortly after, birth $(p=0.001)$ had higher levels of secretory IgA to PT in breast milk at a median of about 58 days post-partum versus women not vaccinated for at least 5 years before delivery [59].

\section{Effectiveness Measures}

In infants aged $<2$ or $<3$ months, the effectiveness of pertussis immunization during pregnancy ranges from 53 to $93 \%$ in preventing pertussis (Table 2) [60-67]. In addition, Tdapduring pregnancy reduces pertussis-related hospitalization in infants, with vaccine effectiveness estimated to be $58-94 \%$ $[65,66,68]$. Among hospitalized infants with pertussis, hospital stays were shorter in those whose mothers were vaccinated versus those of unvaccinated mothers (median 3 vs. 6 days; $p=0.02$ ) and, notably, no infants of vaccinated mothers had seizures, required intubation, or died [68]. However, a much smaller study reported that the duration of hospitalization did not differ between infants whose mothers received pertussis vaccination and those of unvaccinated mothers (median 4 vs. 3.5 days; $p=0.58$ ) [63]. Vaccine effectiveness against pertussis-related death was estimated to be $95 \%$ in one UK-based study [61].

Vaccine effectiveness was affected by the time between vaccination and delivery, and the gestational week at vaccination. Indeed, effectiveness appeared higher if maternal vaccination occurred during gestational weeks 27-36 than vaccination outside this period during pregnancy [66, 67]. No reduction in pertussis rates were observed in infants of mothers who received Tdap at $<27$ gestational weeks in one study with infant follow-up to 18 months [69]. The protection afforded by vaccination prior to pregnancy or post-partum also appears at best low $[62,66]$. In the UK program of maternal immunization, effectiveness was similar in infants aged $<2-3$ months whose mothers were vaccinated 7-27 days before delivery and in those whose mothers were vaccinated earlier in pregnancy ( $\geq 28$ days before delivery), but lower in those whose mothers were vaccinated between 6 days before and 13 days after delivery (91\% vs. $91 \%$ vs. $38-43 \%$, respectively) $[60,61]$.

Three studies investigated the clinical impact of potential immunological blunting of the infant's response to their primary immunization series [61, 62, 70]. Two studies reported that the relative effectiveness of maternal immunization after each of the three doses of the primary series waned but remained positive, indicating the absence of a negative effect of maternal immunization on the protection afforded by the primary immunization, regardless of any immunological blunting (Table 2 $[61,62])$. In the third study, which followed 408 infants of women vaccinated during pregnancy in New Zealand (94.2-98.5\% received each of the three infant DTaP doses on time), no infant developed pertussis over a period of up to 12 months despite nine infants having contact with a confirmed case, and there being a high rate of pertussis in the community [70].

\section{DISCUSSION}

The concept of "cocooning" was previously considered an appropriate intervention for preventing disease among infants too young to be vaccinated. Over time, it was realized that the broad vaccination coverage required to achieve acceptable protection of the newborn was unrealistic, at least beyond the parents and siblings. Given the resurgence of pertussis, a number of countries have introduced maternal pertussis vaccinations during pregnancy as a strategy to confer protection in young infants.

Our review confirms that maternal pertussis vaccination during pregnancy elicits robust and durable responses to all vaccine antigens similar to those observed in non-pregnant women. Moreover, maternal immunization results in efficient transplacental transfer of anti-pertussis antibodies to the fetus, demonstrated by higher antibody GMCs in cord blood than maternal blood. Pertussis-specific antibodies persisted in the infant and reduced the risk of disease during the period before childhood pertussis vaccination. Although a minimum interval of 2 weeks between maternal vaccine administration and delivery appears to be required, longer intervals, 
including vaccination during the second trimester or early in the third trimester, lead to higher antibody concentrations in newborn infants and allow for maturation of the immune response, thus improving the quality (i.e., avidity) of antibodies transferred to the fetus [43].

The available evidence suggests that maternal Tdap vaccine effectiveness is maintained during the infant's primary pertussis vaccination series. It is encouraging that no negative impact on the effectiveness of the primary DTaP series was reported. However, maternal pertussis immunization causes a relative blunting of the infants' immune response to pertussis antigens after primary immunization with acellular pertussis-component vaccines, although the specific antigen(s) affected varied across studies. Nevertheless, long term consequences of this blunting effect remain to be determined and may take several years to establish. Another question raised may be the potential consequences of blunting on other diseases and in other settings. In addition to reporting the immunogenicity of pertussis vaccine, a UK study identified blunting of responses to other antigens, particularly diphtheria toxoid or diphtheria protein-conjugated vaccines, although most infants $(97.7 \%$ and $84.4 \%$, respectively) achieved protective antibody concentrations [53]. Short- and long-term clinical consequences need to be ascertained, particularly as the strategy of immunization during pregnancy expands globally and may be introduced in settings where diphtheria remains a public health concern. It is also important to understand whether blunting occurs in infants receiving whole-cell pertussis primary series and whether there are associated short- and longterm consequences.

Additional research will also be needed to clarify whether the findings of this review are applicable to other pertussis vaccines currently in development. Genetically detoxified pertussis toxin-based vaccines are being developed to enhance immunogenicity against PT, both quantitatively and qualitatively. The impact of these vaccines on maternal immunization will need to be studied as part of their research and development. Finally, it should be noted that the overwhelming majority of pregnant women receiving pertussis vaccination were originally primed with whole-cell pertussis vaccines. Whole-cell pertussis vaccine priming induces a robust immune response after subsequent booster doses than does acellular pertussis vaccine priming. It therefore seems reasonable to question, as cohorts of women of child-bearing age shift from whole-cell pertussis vaccine to acellular pertussis vaccine priming, whether their responses to pertussis vaccination in pregnancy will remain robust enough to sufficiently protect the newborn.

Our systematic review has a number of limitations. The heterogeneity in immunological endpoints, the diagnostic assays, seroconversion thresholds, and criteria applied between the studies limit their comparability. In addition, the protective threshold of pertussisspecific antibodies remains to be established, which limits the interpretation of the antibody GMCs presented. A substantial number of studies reviewed were of small sample size, potentially affecting the external validity of the evidence, as well as the power of these studies to detect associations. The restriction to articles published in the English language may have caused some international studies to be overlooked. The strength of this review lies in our adherence to established methods for conducting systematic reviews, including extensive literature searching methods across several databases, and a wide inclusive publication date range. It is reassuring that, despite these limitations, the generally concordant findings between studies supports our conclusions.

There have been four recent reviews assessing the effectiveness and safety of pertussis vaccination in pregnancy [71-74]. Gkentzi et al. [71], McMillan et al. [72] and Furuta et al. [74] all performed reviews of the literature published up to May 2016, and Campbell et al. through to April 2017 [73]. Our review includes several more recently published studies not captured in the earlier reviews, reflecting the high general interest in maternal pertussis vaccination during pregnancy. The findings in the current review and our accompanying safety review (D'Heilly et al., preparation) are in concordance with the earlier reviews. 


\section{CONCLUSION}

In conclusion, this systematic review provides evidence that maternal pertussis immunization with Tdap during pregnancy provides sufficient maternally-derived pertussis antibodies in infants, and protects infants aged $<2$ or $<3$ months against pertussis. Vaccine effectiveness was not adversely affected by the relative blunting of the infants' immune response to some, but not all, pertussis antigens after primary immunization with acellular pertussiscomponent vaccines. However, there remains a need to better characterize the impact of maternal pertussis vaccination, so as to determine how it will evolve as the wider maternal population is exposed to pertussis vaccines during pregnancy, and how it will affect the epidemiology of the disease. Nonetheless, a number of countries now recommend maternal pertussis immunization as part of their overall pertussis control program.

\section{ACKNOWLEDGEMENTS}

Funding. This review and the journal's rapid service fee were funded by Sanofi Pasteur.

Medical Writing and Editorial Assistance. Editorial assistance with the preparation of the manuscript was provided by Caroline Spencer on behalf of inScience Communications, Springer Healthcare, UK, and was funded by Sanofi Pasteur. The authors also thank Burnedette Rose Hill for editorial assistance and manuscript coordination on behalf of SanofiPasteur.

Authorship. All named authors meet the International Committee of Medical Journal Editors (ICMJE) criteria for authorship for this article, take responsibility for the integrity of the work as a whole, and have given their approval for this version to be published.

Disclosures. Charlotte Switzer is an employee of Sanofi Pasteur. Caroline D'Heilly is an employee of Sanofi Pasteur. Denis Macina is an employee of Sanofi Pasteur.

Compliance with Ethics Guidelines. This article is based on previously conducted studies and does not contain any studies with human participants or animals performed by any of the authors.

Open Access. This article is distributed under the terms of the Creative Commons Attribution-NonCommercial 4.0 International License (http://creativecommons.org/licenses/ by-nc/4.0/), which permits any noncommercial use, distribution, and reproduction in any medium, provided you give appropriate credit to the original author(s) and the source, provide a link to the Creative Commons license, and indicate if changes were made.

\section{REFERENCES}

1. Hewlett EL, Edwards KM. Clinical practice. Pertussis-not just for kids. $\mathrm{N}$ Engl J Med. 2005;352(12):1215-22.

2. Celentano LP, Massari M, Paramatti D, Salmaso S, Tozzi AE. Resurgence of pertussis in Europe. Pediatr Infect Dis J. 2005;24(9):761-5.

3. McGirr AA, Tuite AR, Fisman DN. Estimation of the underlying burden of pertussis in adolescents and adults in Southern Ontario, Canada. PLoS ONE. 2013;8(12):e83850.

4. Mink CA, Sirota NM, Nugent S. Outbreak of pertussis in a fully immunized adolescent and adult population. Arch Pediatr Adolesc Med. 1994;148(2):153-7.

5. Rosenthal S, Strebel P, Cassiday P, Sanden G, Brusuelas K, Wharton M. Pertussis infection among adults during the 1993 outbreak in Chicago. J Infect Dis. 1995;171(6):1650-2.

6. Skoff TH, Hadler S, Hariri S. The epidemiology of nationally reported pertussis in the United States, 2000-2016. Clin Infect Dis. 2018.

7. Gutierrez-Trujillo G, Perez-Enriquez LR, GonzalezGarcia A, Coreno-Juarez MO, Ramirez-Rosales G, Grajales-Muniz C. Preventable diseases by vaccination: coverage and impact. Rev Med Inst Mex Seguro Soc. 2006;44(Suppl 1):S97-109. 
8. Pertussis epidemic-Washington (2012) Morb Mortal Wkly Rep. 2012;61(28):517-22.

9. Carlsson RM, von Segebaden K, Bergstrom J, Kling AM, Nilsson L. Surveillance of infant pertussis in Sweden 1998-2012; severity of disease in relation to the national vaccination programme. Euro Surveill. $2015 ; 20(6)$.

10. Haberling DL, Holman RC, Paddock CD, Murphy TV. Infant and maternal risk factors for pertussisrelated infant mortality in the United States, 1999 to 2004. Pediatr Infect Dis J. 2009;28(3):194-8.

11. Public Health England. Laboratory confirmed cases of pertussis reported to the enhanced pertussis surveillance programme in England: annual report 2013. 2014.

12. Straney L, Schibler A, Ganeshalingham A, Alexander J, Festa M, Slater A, et al. Burden and outcomes of severe pertussis infection in critically ill infants. Pediatr Crit Care Med. 2016;17(8):735-42.

13. Winter K, Harriman K, Zipprich J, Schechter R, Talarico J, Watt J, et al. California pertussis epidemic, 2010. J Pediatr. 2012;161(6):1091-6.

14. Bisgard KM, Pascual FB, Ehresmann KR, Miller CA, Cianfrini $\mathrm{C}$, Jennings $\mathrm{CE}$, et al. Infant pertussis: who was the source? Pediatr Infect Dis J. 2004;23(11):985-9.

15. Wendelboe AM, Njamkepo E, Bourillon A, Floret DD, Gaudelus J, Gerber M, et al. Transmission of Bordetella pertussis to young infants. Pediatr Infect Dis J. 2007;26(4):293-9.

16. Skoff TH, Kenyon C, Cocoros N, Liko J, Miller L, Kudish K, et al. Sources of infant pertussis infection in the United States. Pediatrics. 2015;136(4):635-41.

17. Abu-Raya B, Bettinger JA, Vanderkooi OG, Vaudry W, Halperin SA, Sadarangani M. Burden of children hospitalized with pertussis in Canada in the acellular pertussis vaccine era, 1999-2015. J Pediatr Infect Dis Soc. 2018.

18. Frere J, De Wals P, Ovetchkine P, Coic L, Audibert F, Tapiero B. Evaluation of several approaches to immunize parents of neonates against B pertussis. Vaccine. 2013;31(51):6087-91.

19. Lim GH, Deeks SL, Crowcroft NS. A cocoon immunisation strategy against pertussis for infants: does it make sense for Ontario? Euro Surveill. 2014;19(5):20688.

20. Rossmann Beel E, Rench MA, Montesinos DP, Healy CM. Acceptability of immunization in adult contacts of infants: possibility of expanding platforms to increase adult vaccine uptake. Vaccine. 2014;32(22):2540-5.

21. Simonetti A, Martini I, Bonomo G, D'Avino R, Puggina $\mathrm{P}$, Vairo U, et al. Improving adherence rates to a cocooning program: a pilot experience in Italy. Hum Vaccin Immunother. 2013;9(5):1142-5.

22. Skowronski DM, Janjua NZ, Tsafack EP, Ouakki M, Hoang L, De Serres G. The number needed to vaccinate to prevent infant pertussis hospitalization and death through parent cocoon immunization. Clin Infect Dis. 2012;54(3):318-27.

23. Urwyler P, Heininger U. Protecting newborns from pertussis-the challenge of complete cocooning. BMC Infect Dis. 2014;14:397.

24. Moher D, Liberati A, Tetzlaff J, Altman DG. Preferred reporting items for systematic reviews and meta-analyses: the PRISMA statement. PLoS Med. 2009;6(7):e1000097.

25. Switzer C, Macina D. Safety and effectiveness of pertussis immunization during pregnancy. Available at: http://www.crd.york.ac.uk/PROSPERO/ display_record.php?ID=CRD42016038317. Last accessed 16 February 2019.

26. O'Connor D, Green S, Higgins JPT. Chapter 5: Defining the review question and developing criteria for including studies. Cochrane handbook for systematic reviews of interventions. Version 5.0.0 ed2008 ed2008.

27. Abu Raya B, Srugo I, Kessel A, Peterman M, Bader D, Gonen R, et al. The effect of timing of maternal tetanus, diphtheria, and acellular pertussis (Tdap) immunization during pregnancy on newborn pertussis antibody levels-a prospective study. Vaccine. 2014;32(44):5787-93.

28. Abu Raya B, Srugo I, Kessel A, Peterman M, Vaknin A, Bamberger E. the decline of pertussis-specific antibodies after tetanus, diphtheria, and acellular pertussis immunization in late pregnancy. J Infect Dis. 2015;212(12):1869-73.

29. Fallo AA, Neyro SE, Manonelles GV, Lara C, Hozbor $D$, Zintgraff $J$, et al. Prevalence of pertussis antibodies in maternal blood, cord serum, and infants from mothers with and those without Tdap booster vaccination during pregnancy in Argentina. J Pediatric Infect Dis Soc. 2018;7(1):11-7.

30. Gandhi M, Devaraj S, Sangi-Haghpeykar H, Mastrobattista J. The effect of body mass index on postvaccination maternal and neonatal pertussis antibody levels. J Reprod Immunol. 2015;112:34-7.

31. Gray L, Quataert S, Secor-Socha S, Thornburg L, Grzesik K, Muthukrishnan G, et al. Antibody 
responses to pertussis vaccination in pregnant versus non-pregnant subjects. Reprod Sci. 2016;23(Suppl 1):82A.

32. Halperin SA, Langley JM, Ye L, MacKinnonCameron D, Elsherif M, Allen VM, et al. A randomized controlled trial of the safety and immunogenicity of tetanus, diphtheria, and acellular pertussis vaccine immunization during pregnancy and subsequent infant immune response. Clin Infect Dis. 2018;67(7):1063-71.

33. Hardy-Fairbanks AJ, Pan SJ, Decker MD, Johnson $\mathrm{DR}$, Greenberg DP, Kirkland KB, et al. Immune responses in infants whose mothers received Tdap vaccine during pregnancy. Pediatr Infect Dis J. 2013;32(11):1257-60.

34. Hincapie-Palacio D, Hoyos MC, Ochoa J, Montoya $\mathrm{N}$, Garcia D, Osorio E. Effect of maternal immunization against pertussis in Medellin and the metropolitan area, Colombia, 2016-2017. Vaccine. 2018;36(27):3984-91.

35. Hoang HT, Leuridan E, Maertens K, Nguyen TD, Hens $\mathrm{N}, \mathrm{Vu} \mathrm{NH}$, et al. Pertussis vaccination during pregnancy in Vietnam: results of a randomized controlled trial of pertussis vaccination during pregnancy. Vaccine. 2016;34(1):151-9.

36. Huygen K, Cabore RN, Maertens K, Van Damme P, Leuridan E. Humoral and cell mediated immune responses to a pertussis containing vaccine in pregnant and nonpregnant women. Vaccine. 2015;33(33):4117-23.

37. Maertens K, Hoang TT, Nguyen TD, Cabore RN, Duong TH, Huygen K, et al. The effect of maternal pertussis immunization on infant vaccine responses to a booster pertussis-containing vaccine in Vietnam. Clin Infect Dis. 2016;63(suppl 4):S197-204.

38. Munoz FM, Bond NH, Maccato M, Pinell P, Hammill HA, Swamy GK, et al. Safety and immunogenicity of tetanus diphtheria and acellular pertussis (Tdap) immunization during pregnancy in mothers and infants: a randomized clinical trial. JAMA. 2014;311(17):1760-9.

39. Vilajeliu A, Gonce A, Lopez M, Costa J, Rocamora L, Rios J, et al. Combined tetanus-diphtheria and pertussis vaccine during pregnancy: transfer of maternal pertussis antibodies to the newborn. Vaccine. 2015;33(8):1056-62.

40. Villarreal Pérez JZ, Ramírez Aranda JM, de la O Cavazos M, Zamudio Osuna MJ, Perales Dávila J, Ballesteros Elizondo MR, et al. Randomized clinical trial of the safety and immunogenicity of the Tdap vaccine in pregnant Mexican women. Hum Vaccin Immunother. 2017;13(1):128-35.
41. Healy CM, Rench MA, Baker CJ. Importance of timing of maternal combined tetanus, diphtheria, and acellular pertussis (Tdap) immunization and protection of young infants. Clin Infect Dis. 2013;56(4):539-44.

42. Fortner KB, Swamy GK, Broder KR, Jimenez-Truque $\mathrm{N}$, Zhu Y, Moro PL, et al. Reactogenicity and immunogenicity of tetanus toxoid, reduced diphtheria toxoid, and acellular pertussis vaccine (Tdap) in pregnant and nonpregnant women. Vaccine. 2018;36(42):6354-60.

43. Abu Raya B, Bamberger E, Almog M, Peri R, Srugo I, Kessel A. Immunization of pregnant women against pertussis: the effect of timing on antibody avidity. Vaccine. 2015;33(16):1948-52.

44. Gall SA, Myers J, Pichichero M. Maternal immunization with tetanus-diphtheria-pertussis vaccine: effect on maternal and neonatal serum antibody levels. Am J Obstet Gynecol. 2011;204(4):334.

45. Healy CM, Rench MA, Swaim LS, Smith EOB, SangiHaghpeykar $\mathrm{H}$, Mathis $\mathrm{MH}$, et al. Association between third-trimester Tdap immunization and neonatal pertussis antibody concentration. JAMA. 2018;320(14):1464-70.

46. Maertens K, Cabore RN, Huygen K, Hens N, Van Damme $\mathrm{P}$, Leuridan E. Pertussis vaccination during pregnancy in Belgium: results of a prospective controlled cohort study. Vaccine. 2016;34(1):142-50.

47. Naidu M, Wallace E, Giles M. Pertussis vaccine: when is the optimal time to vaccinate during pregnancy? Br J Obstet Gynaecol. 2015;122:365.

48. Wanlapakorn N, Maertens K, Chaithongwongwatthana S, Srimuan D, Suratannon N, Vongpunsawad $S$, et al. Assessing the reactogenicity of Tdap vaccine administered during pregnancy and antibodies to Bordetella pertussis antigens in maternal and cord sera of Thai women. Vaccine. 2018;36(11):1453-9.

49. Abraham C, Pichichero M, Eisenberg J, Singh SI. Third-trimester maternal vaccination against pertussis and pertussis antibody concentrations. Obstet Gynecol. 2018;131(2):364-9.

50. Eberhardt CS, Blanchard-Rohner G, Lemaitre B, Boukrid M, Combescure C, Othenin-Girard V, et al. Maternal immunization earlier in pregnancy maximizes antibody transfer and expected infant seropositivity against pertussis. Clin Infect Dis. 2016;62(7):829-36.

51. Eberhardt CS, Blanchard-Rohner G, Lemaitre B, Combescure C, Othenin-Girard V, Chilin A, et al. Pertussis antibody transfer to preterm neonates 
after second- versus third-trimester maternal immunization. Clin Infect Dis. 2017;64(8):1129-32.

52. Vilajeliu A, Ferrer L, Munros J, Gonce A, Lopez M, Costa J, et al. Pertussis vaccination during pregnancy: antibody persistence in infants. Vaccine. 2016;34(33):3719-22.

53. Ladhani SN, Andrews NJ, Southern J, Jones CE, Amirthalingam G, Waight PA, et al. Antibody responses after primary immunization in infants born to women receiving a pertussis-containing vaccine during pregnancy: single arm observational study with a historical comparator. Clin Infect Dis. 2015;61(11):1637-44.

54. Kent A, Ladhani SN, Andrews NJ, Matheson M, England A, Miller E, et al. Pertussis antibody concentrations in infants born prematurely to mothers vaccinated in pregnancy. Pediatrics. 2016;138(1).

55. Cabore RN, Maertens K, Dobly A, Leuridan E, Van Damme P, Huygen K. Influence of maternal vaccination against diphtheria, tetanus, and pertussis on the avidity of infant antibody responses to a pertussis containing vaccine in Belgium. Virulence. 2017;8(7):1245-54.

56. Maertens K, Hoang TT, Nguyen TD, Caboré RN, Duong TH, Huygen K, et al. The effect of maternal pertussis immunization on infant vaccine responses to a booster pertussis-containing vaccine in Vietnam. Clin Infect Dis. 2016;63(Suppl 4):S197-204.

57. Maertens K, Cabore RN, Huygen K, Vermeiren S, Hens N, Van Damme P, et al. Pertussis vaccination during pregnancy in Belgium: follow-up of infants until 1 month after the fourth infant pertussis vaccination at 15 months of age. Vaccine. 2016;34(31):3613-9.

58. Abu Raya B, Srugo I, Kessel A, Peterman M, Bader D, Peri R, et al. The induction of breast milk pertussis specific antibodies following gestational tetanusdiphtheria-acellular pertussis vaccination. Vaccine. 2014;32(43):5632-7.

59. De Schutter S, Maertens K, Baerts L, De Meester I, Van Damme P, Leuridan E. Quantification of vaccine-induced antipertussis toxin secretory IgA antibodies in breast milk: comparison of different vaccination strategies in women. Pediatr Infect Dis J. 2015;34(6):e149-52.

60. Amirthalingam G, Andrews N, Campbell H, Ribeiro S, Kara E, Donegan K, et al. Effectiveness of maternal pertussis vaccination in England: an observational study. Lancet. 2014;384(9953):1521-8.

61. Amirthalingam G, Campbell H, Ribeiro S, Fry NK, Ramsay M, Miller E, et al. Sustained effectiveness of the maternal pertussis immunization program in
England 3 years following introduction. Clin Infect Dis. 2016;63(suppl 4):S236-43.

62. Baxter R, Bartlett J, Fireman B, Lewis E, Klein NP. Effectiveness of vaccination during pregnancy to prevent infant pertussis. Pediatrics. 2017;139(5).

63. Dabrera G, Amirthalingam G, Andrews N, Campbell H, Ribeiro S, Kara E, et al. A case-control study to estimate the effectiveness of maternal pertussis vaccination in protecting newborn infants in England and Wales, 2012-2013. Clin Infect Dis. 2015;60(3):333-7.

64. Romanin V, Acosta AM, Sagradini S, Briere E, Sanchez SM, Sevilla ME, et al. Effectiveness of Tdap vaccination during pregnancy in preventing infant pertussis in a country with whole-cell pertussis vaccines during childhood: preliminary results of a case-control study in Argentina. Open Forum Infect Dis. 2015;2(Suppl 1):S447.

65. Saul N, Wang K, Bag S, Baldwin H, Alexander K, Chandra $M$, et al. Effectiveness of maternal pertussis vaccination in preventing infection and disease in infants: the NSW public health network case-control study. Vaccine. 2018;36(14):1887-92.

66. Skoff TH, Blain AE, Watt J, Scherzinger K, McMahon M, Zansky SM, et al. Impact of the US maternal tetanus, diphtheria, and acellular pertussis vaccination program on preventing pertussis in infants $<2$ months of age: a case-control evaluation. Clin Infect Dis. 2017;65(12):1977-83.

67. Winter K, Nickell S, Powell M, Harriman K. Effectiveness of prenatal versus post-partum tetanus, diphtheria, and acellular pertussis vaccination in preventing infant pertussis. Clin Infect Dis. 2017;64(1):3-8.

68. Winter K, Cherry JD, Harriman K. Effectiveness of prenatal tetanus, diphtheria, and acellular pertussis vaccination on pertussis severity in infants. Clin Infect Dis. 2017;64(1):9-14.

69. Becker-Dreps S, Butler AM, McGrath LJ, Boggess KA, Weber DJ, Li D, et al. Effectiveness of prenatal tetanus, diphtheria, acellular pertussis vaccination in the prevention of infant pertussis in the US. Am J Prev Med. 2018;55(2):159-66.

70. Walls T, Graham P, Petousis-Harris H, Hill L, Austin $\mathrm{N}$. Infant outcomes after exposure to Tdap vaccine in pregnancy: an observational study. BMJ Open. 2016;6(1):e009536.

71. Gkentzi D, Katsakiori P, Marangos M, Hsia Y, Amirthalingam G, Heath PT, et al. Maternal vaccination against pertussis: a systematic review of the recent literature. Arch Dis Child Fetal Neonatal Ed. 2017;102(5):F456-63. 
72. McMillan M, Clarke M, Parrella A, Fell DB, Amirthalingam G, Marshall HS. Safety of tetanus, diphtheria, and pertussis vaccination during pregnancy: a systematic review. Obstet Gynecol. 2017;129(3):560-73.

73. Campbell H, Gupta S, Dolan GP, Kapadia SJ, Kumar Singh A, Andrews N, et al. Review of vaccination in pregnancy to prevent pertussis in early infancy. J Med Microbiol. 2018;67(10):1426-56.
74. Furuta M, Sin J, Ng ESW, Wang K. Efficacy and safety of pertussis vaccination for pregnant women-a systematic review of randomised controlled trials and observational studies. BMC Pregnancy Childbirth. 2017;17(1):390. 